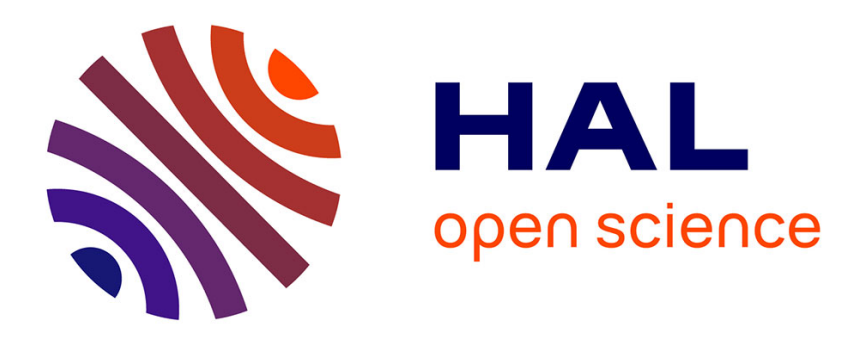

\title{
Networks and dynamical systems
}

\author{
Vadim A. A. Malyshev
}

\section{To cite this version:}

Vadim A. A. Malyshev. Networks and dynamical systems. [Research Report] RR-1468, INRIA. 1991. inria-00075094

\section{HAL Id: inria-00075094 https://hal.inria.fr/inria-00075094}

Submitted on 24 May 2006

HAL is a multi-disciplinary open access archive for the deposit and dissemination of scientific research documents, whether they are published or not. The documents may come from teaching and research institutions in France or abroad, or from public or private research centers.
L'archive ouverte pluridisciplinaire HAL, est destinée au dépôt et à la diffusion de documents scientifiques de niveau recherche, publiés ou non, émanant des établissements d'enseignement et de recherche français ou étrangers, des laboratoires publics ou privés. 


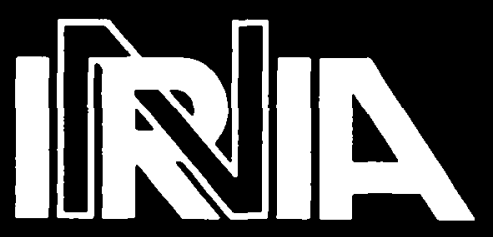

UNITÉ DE RECHERCHE INRIA-ROCQUENCOURT

\section{Rapports de Recherche}

\author{
$N^{\circ} 1468$
}

\section{Programme 1}

Architectures parallèles, Bases de données, Réseaux et Systèmes distribués

\section{NETWORKS AND DYNAMICAL SYSTEMS}

Vadim A. MALYSHEV

Domaine de Voluceau

Rocquencourt

$$
\text { B.P.105 }
$$

78153 Le Chesnay Cedex

$$
\text { France }
$$




\title{
Réseaux et systèmes dynamiques.
}

\author{
Vadim Malyshev
}

\section{Résumé.}

On introduit une nouvelle approche au probleme de la classification des marches aléatoires dans $Z_{+}^{N}$, ainsi que des réseaux de files d'attente avec clients identiques. Elle s'appuie sur l'analyse du système dynamique associé à toute marche aléatoire. D'anciens résultats, en dimension petite, sont présentés avec ce nouveau point de vue. Nous prouvons des résultats nouveaux, pour les dimensions plus grandes, en nous appuyant sur l'existence d'une mesure invariante continue pour le système dynamique sous-jacent. On montre l'importance de deux constantes : l'énergie libre $M$ (le système est ergodique pour $M<0$ ), l'exposant de Lyapounov $L$ (le système est recurrent pour $L<0$ ). On présente des conjectures générales, des exemples, des problèmes ouverts et des liaisons surprenantes avec la théorie ergodique et les perturbations aléatoires des systèmes dynamiques. La notion, fort utile, de perturbation aléatoire "ajustée" s'introduit de façon naturelle.

Addresse : INRIA - Domaine de Voluceau, Rocquencourt, BP105 - 78153 - Le Chesnay. France.

On leave of absence from Moscow State University, Moscow, 119899, USSR. 


\title{
Networks and dynamical systems.
}

\author{
V.A. Malyshev
}

Abstract.

A new approach to the problem of classification of random walks in $\mathbf{z}_{+}^{N}$ or queueing networks with identical customers is introduced. It is based on the analysis of the intrinsic dynamical system associated with the random walk. Earlier results for small dimensions are presented from the novel point of view. We give proofs of new results for higher dimensions related to the existence of continuous invariant measure for the underlying dynamical system. Two constants are shown to be important : the free energy $M<O$ corresponds to ergodicity, the Lyapounov exponent $L<O$ defines recurrence. General conjectures, examples, unsolved problems and surprising connections with ergodic theory, classical dynamical systems and their random perturbations are largely presented. A useful notion naturally arises, the so called scaled random perturbation of a dynamical system.

Postal address : INRIA - Domaine de Voluceau, Rocquencourt, BP105 - 78153 - Le Chesnay . France.

On sabbatical leave from Moscow State University, Moscow, 119899, USSR. Invited by the project MEVAL. 
Contents.

Introduction

\section{CONSTRUCTION OF THE DYNAMICAL SYSTEM}

1.1. Equivalence between identical customer Markov networks with interaction and random walks in $\mathbf{Z}_{+}^{N}$.

1.2. Random walks in $\mathbb{Z}_{+}^{N}$ : main definitions. Second vector field.

1.3. Classification of paths

1.4. Associated dynamical systems

1.5. Multiplicative suspensions over dynamical systems

1.6. About scaled random suspensions

1.7 Scattering with finite exit boundary

2. ACYCLICITY AND SMALL DIMENSIONS

2.1. Dimensions 1, 2, 3 and acyclic random walks

2.2. Scattering in 2-dimensional complexes.

2.3. Dimension 4 and Poincare-Bendixson theory.

\section{UNIFORM CONVERGENCE}

3.1. Unique ergodicity

3.2. Uniformly convergent dynamical systems with many ergodic components

3.3. Transience for uniformly absorbing attractors.

4. MORE DELICATE PHENOMENA

4.1. Liaisons dangereuses.

4.2. Null recurrent essentially deterministic random walks : Gibbs measures.

4.3. Continuous exit boundary.

4.4. Nonstationary effects and zero drifts.

4.5. Continuity, analyticity, CLT.

APPENDIX. ABOUT SOME RELATED GENERAL PROBABILISTIC PROBLEMS CONCLUSION

REFERENCES 


\section{INTRODUCTION : WHY FOR AND WHAT ABOUT?}

First of all, this paper is not a review on networks. There are some mathematical reviews now (see e.g. $[39,40]$ ). Taking one class of networks and only one problem (the stability problem), I try to say everything known to me about it. A very popular and widely accepted extreme point in queueing theory is that papers must have a simple answer and practical advertisement. I pursue here the opposite extreme goal. Why random walks in $\mathbf{Z}_{+}^{N}$ are of primary interest ? There are several reasons :

They describe many practically interesting networks, see section 1.1 , and the methods about which we speak here could also be useful for networks with nonidentical customers. It is not only a problem of probabilistic interest but also a large store of examples. It is closely connected with other branches of mathematics : the classification problem for random walks in $\mathbf{Z}_{+}^{N}$ is a probabilistic version of the well known problems in functional analysis and partial differential equations : when is multidimensional Toeplitz or other elliptic operator in $\mathbf{Z}_{+}^{N}$ is invertible ? It has also much in common with the behaviour of diffusion processes near non smooth boundary of large codimension. As it will be shown here, such random walks are closely connected with classical dynamical systems and their random perturbations.

The main goals and peculiarities of this paper are the following ones:

1. Presentation of the new approach and a review of previous results from a novel unified point of view ;

2. Like for any honorable mathematical theory, many general problems with the predicate $\forall$ are hopelessly unsolvable (I try to show this) and a theory lives by providing many new examples, classes of solvable problems, ideas and looking for clever questions. I try to mention all examples known to me.

3. Many examples are really new. So there was no space to penetrate more deeply to most of them. And moreover one cannot be sure enough that one network is more important than the other one. It seemed to me that it was more important now to explain the general situation than to get separate results.

4. Exact formulations are given, but some proofs are omitted which are clear modulo being familiar with the previous results. If I did not know how to prove some results or if it could take more time then I formulated it as a problem.

5. I tried to emphasize great possible influence of the classical ergodic theory on networks. I hope also that the notion of scaled random suspension could be useful in other fields. 
I had discussions with G. Fayolle, I. Ignatyuk and M. Menshikov but all eventual mistakes are of my own. I am also thankful to Yu. Kifer, V. Oseledec, Ya. Sinai, S. Smirnov, for sending me their papers, useful comments and discussions. 


\section{CONSTRUCTION OE THE DYNAMICAL SYSTEM}

1.1 Equivalence between identical customer Markov networks with interaction and random walks in $\mathbf{Z}_{+}^{N}$.

We want to recall some definitions from [2]. Let us consider a discrete time homogeneous Markov chain $\mathscr{L}$ which is assumed to be irreducible and aperiodic unless otherwise stated. The set of states is $z_{+}^{N}=\left\{\left(z, \ldots, z_{N}\right): z_{i} \geq 0\right.$ are integers $\}, p_{\alpha \beta}^{k}$ are $k-$ step transition probabilities on $\mathbb{L}, M^{k}(\alpha)=\left(M_{l}^{k}(\alpha), \ldots, M_{N}^{k}(\alpha)\right)$ be the vector of mean jump from the point $\alpha$ in $k$ steps; $p_{\alpha \beta}^{I}=p_{\alpha \beta}, M^{1}(\alpha)=M(\alpha)$.

For any $\Lambda \subset\{1,2, \ldots, N\}$ we define the face $B^{\Lambda}$ of $\mathbb{R}_{+}^{N}=\left\{\left(r_{1}, \ldots, r_{N}\right\}: r_{i} \geqslant 0\right.$ are real $\}$ by

$$
B^{\Lambda}=\left\{\left(r_{1}, \ldots, r_{N}\right): r_{i}>0, i \in \Lambda ; r_{i}=0, i \in \Lambda\right\}
$$

Sometimes we shall write $\Lambda$ instead of $B^{\Lambda}$ whenever confusion cannot occur.

We consider only random walks satisfying

Condition $A_{1}$ (boundedness of jumps):

$$
p_{\alpha \beta}=0 \text { for }\|\alpha-\beta\|>d \text {, }
$$

where $d>0$ and $\|\alpha\|=\max _{i} \mid \alpha_{i} /, \alpha=\left(\alpha_{l}, \ldots, \alpha_{N}\right)$.

Condition $\mathrm{A}_{2}$ (homogeneity):

for any $\Lambda$ and for any $a \in B^{\Lambda} \cap \mathbb{Z}_{+}^{N}$,

$$
p_{\alpha \beta}=p_{\alpha+a, \beta+a}
$$

for all $\alpha \in B^{\Lambda} \cap \mathbb{Z}_{+}^{N}, \beta \in \mathbb{Z}_{+}^{N}$.

We define the first vector field on $R_{+}^{N}$ to be constant on any $B^{\Lambda}$ and equal to

$$
M_{\Lambda} \equiv M(\alpha), \alpha \in \Lambda
$$

We shall sometimes consider more general class of random walks (it appears in [29 ]) defined by 
Condition $B_{2}$ (smoothness): let for any $B^{\Lambda}$ and any integer component vector $a=$ $\left(a_{1}, \ldots, a_{N}\right)$ such that $\|a\| \leqslant \delta$ and $a_{i} \geq 0$ for $i \in \Lambda$, be given a smooth nonnegative function $p_{a}(\alpha), \alpha \in B^{\Lambda},\|\alpha\|=1$, such that $\Sigma_{a} p_{a}(\alpha)=1$ for all $\alpha$. Then we assume that

$$
p_{\alpha, \alpha+a} \equiv p_{a}\left(\frac{\alpha}{\|\alpha\|} \quad\right. \text { outside some finite set. }
$$

Let us note that in general $p_{a}(\alpha)$ is not even continuous at the boundaries of faces.

Unless otherwise stated we assume that conditions $A_{1}$ and $A_{2}$ hold.

Now we consider a class of networks with $N$ queues.All customers in the same queue are considered to be identical (no types, no marks) and so a state of the network at time $t$ is completely defined by the vector

$$
\alpha=\left(n_{1}(t), \ldots, n_{N}(t)\right)
$$

where $n_{i}$ is the number of customers in queue $i$.

One can have different transitions

$$
\alpha=\left(n_{1}(t), \ldots n_{N}(t)\right) \rightarrow \beta=\left(n_{1}(t)+i_{1}, \ldots, n_{N}(t)+i_{N}\right)
$$

with intensities $\lambda_{\alpha \beta}$. Different synchronisation constraints (see discussion in [1]) can give different possible values of the vector

$$
\beta-\alpha=\left(i_{1}, \ldots i_{N}\right)
$$

with nonzero $\lambda_{\alpha \beta}$. E.g. for continuous time Jackson network not more than two components of the vector (1) can be different from zero. But joint arrival and joint service can give nonzero $\lambda_{\alpha \beta}$. to any value of the vector (1).

As usual we define the corresponding r.w. for $w$ sufficiently small by

$$
p_{\alpha \beta}=w \lambda_{\alpha \beta}, \alpha \neq \beta, p_{\alpha \alpha}=1-\sum_{\beta \neq \alpha} p_{\alpha \beta} .
$$

This class of networks is not sufficient to get arbitrary r.w. of the class $A_{1,2}$ because transition intensities on the faces are restrictions of transition intensities inside $\mathbb{Z}_{+}^{N}$. But if we add also interaction between nodes we get any r.w. from that class. The interaction means that $\lambda_{\alpha \beta}$ depend also on which nodes of the network are empty.More exactly 
$\lambda_{\alpha \beta \cdot}=\lambda_{\alpha \beta} \cdot(\Lambda ; \beta-\alpha)$

which means that $\lambda_{\alpha \beta}$. is a function of $\beta-\alpha$ and of the face $\Lambda$ to which $\alpha$ belongs.

So these networks can provide us any Markov chain with state space $z_{+}^{N}$ of the class $A_{1,2}$.

The examples of such networks are : Jackson (see references in [ 3 ] ), buffered ALOHA, $N$ coupled-processors [ 13 ] etc. A new class of networks for data basis is considered in [12].

Problem 1.1.1. Is it possible to get simpler ergodicity conditions for networks without interaction but with different synchronisation constraints?

We examine here the problem of classification of such chains. For $N=1,2,3,4$ now we have general sufficiently complete results (see [ 7-12,2] ). Our goal here is to show that in general to obtain necessary and sufficient conditions for $N \geqslant 5$ leads to substantial problems in classical dynamical systems and ergodic theory. General philosophy behind this is the following. For some practically interesting systems there are simpler methods to get complete or partial results (see e.g. [3] , [13] ). But nevertheless even from the practical point of view one needs general understanding of the situation to foresee the possibilities of research .

Networks where customers can have different types and marks could also be considered from this point of view. But here the work is only at the starting point ([15], [14] ) even for small number of queues.

\subsection{Random walks in $\mathbf{Z}_{+}^{\mathrm{N}}$ : main definitions.Second vector field.}

Induced chains. For any $\Lambda \neq\{1, \ldots, N\}$ we choose an arbitrary point $a \in B^{\Lambda} \cap \mathbf{Z}_{+}^{N}$ and draw a plane $C^{\Lambda}$ of dimension $N-/ \Lambda /$ perpendicular to $B^{\Lambda}$ and containing $a$. We define the induced Markov chain $\mathscr{L}^{\Lambda}$ with the state space $C^{\Lambda}$ and transition probabilities

$$
\Lambda_{\alpha \beta}=p_{\alpha \beta}+\sum_{\gamma \neq \beta} p_{\alpha \gamma}, \alpha, \beta \in C^{\Lambda}
$$

where summation is performed over all $\gamma \in \mathbb{Z}_{+}^{N}$ such that the straight line connecting $\gamma$ and $\beta$ is perpendicular to $C A$.

In this paper we shall make a series of assumptions $O_{i}, i=1,2, \ldots$. All of them hold for all points in parameter space except for some hypersurfaces of lower dimension.More exactly we define the parameter space $P=P_{d}$ depending on the constant $d$ which is assumed to be fixed. Due to conditions $A_{1,2}$ it is a product of several simplexes in finitedimensional euclidean space. 
Assumption $O_{1}$. For any $\Lambda$ the chain $\mathcal{L}^{\Lambda}$ is irreducible and aperiodic.

We call $B^{\Lambda}$ (or $\Lambda$ ) ergodic if $\mathfrak{L}^{\Lambda}$ is ergodic. For an ergodic $\mathfrak{L}^{\Lambda}$ let $\pi^{\Lambda}(\eta), \gamma \in C^{\Lambda}$, be its stationary transition probabilities. We introduce the vector $v^{\Lambda}=\left(v_{1}, \ldots,\left(v_{N}^{\Lambda}\right)\right.$ by setting

$$
\begin{gathered}
v_{i}^{\Lambda}=0, i \in \Lambda, \\
v_{i}^{\Lambda}=\Sigma_{\gamma \in C^{\Lambda}} \pi^{\Lambda}(\gamma) M_{i}(\gamma), i \in \Lambda .
\end{gathered}
$$

For $\Lambda=\{1, \ldots, N\}$ we call $\Lambda$ ergodic by definition and put

$$
v^{\Lambda} \equiv M(\alpha), \alpha \in B^{\Lambda} \cap \mathbf{z}_{+}^{N}
$$

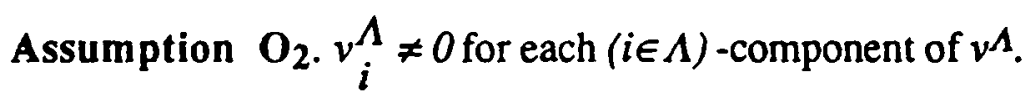

Problem 1.2.1. Homogeneity and boundedness of jumps conditions can be essentially weakened. As in [2] one can consider different probabilities of jumps from the sets $B_{c}^{\Lambda} \cap \mathbb{Z}_{+}^{N}$, where

$$
B_{c}^{\Lambda}=\left\{\left(r_{1}, \ldots, r_{N}\right): r_{i}>c, i \in \Lambda ; r_{i}=c, i \notin \Lambda\right\}, 0 \leqslant c \leqslant 0,
$$

and also jumps which are bounded only from below but first and second vector field are assumed to exist. Induced chains and second vector field are defined here in the same way. I do not know any example where results and ideas could change under such type of generalisations. Proofs however can become more involved. It is important to achieve good understanding when such examples could appear.

Ingoing, outgoing and neutral faces.

Let us fix $\Lambda, \Lambda_{1}$ so that $\Lambda \supset \Lambda_{1}, \Lambda \neq \Lambda_{1}$, then $\bar{B} \Lambda \supset B^{\Lambda_{1}}\left(\bar{B} \Lambda\right.$ is the closure of $\left.B^{\Lambda}\right)$. Let $B^{\Lambda}$ be ergodic and so $v^{\Lambda}$ is defined. There are three possibilities of the direction of $v^{\Lambda}$ w.r.t. $B \Lambda_{1}$.

We say that $B^{\Lambda}$ is an ingoing (outgoing) face for $B^{\Lambda}$ if all the coordinates $v_{i}^{\Lambda}$ for $i \in \Lambda$ $\Lambda_{1}$ are negative(positive). Otherwise we say that $B^{\Lambda}$ is neutral.

As an example we give simple sufficient criteria for a face to be ergodic.

Proposition 1.2.1. A face $B^{\Lambda}$ of dimension $N-1$ is ergodic iff $v_{1}^{\{1, \ldots, N\}}<0$ for $i$ $\epsilon\{1, \ldots, N\}-\Lambda$.

Proposition 1.2.2. If all faces $B^{\Lambda}$ with $\Lambda \supset \Lambda_{1}$ are ergodic and ingoing for $B^{\Lambda}$ then $B^{A_{1}}$ is ergodic.

We leave the proof to the reader using the main result of [2]. 
Second vector field. To any point $x \in R_{+}^{N}$ we assign a vector $v(x)$ and call this function a second vector field. It can be multivalued on some nonergodic faces. We put for ergodic faces $B^{\Lambda}$

$$
v(x) \equiv v^{\Lambda}, x \in B^{\Lambda}
$$

if $B^{\Lambda}$ is nonergodic then at any point $x \in B^{\Lambda} \quad v(x)$ takes all values $v^{\Lambda}$ for which $B^{\Lambda}$ is an outgoing face w.r.t. $B^{\Lambda}$. In other words for $x$ belonging to nonergodic faces (with $|x|$ sufficiently large)

$$
x+v(x) \in \mathbb{R}_{+}^{N}
$$

for any value $v(x)$. If there is no such vectors we put $v(x)=0$ for $x \in B^{\Lambda} 1$.Points $x \in$ $\mathbb{R}_{+}^{N}$ where $v(x)$ is more than one-valued are called branch points.

There are few interesting examples for which one needs only the first vector field to obtain ergodicity conditions for a random walk but it is nevertheless the case for Jackson networks [3]. In the general case the second vector field is indispensable to consider.

The following proposition demonstrates usefulness of the second vector field.

Proposition 1.2.3. If for some ergodic face $\Lambda$ all components of $v^{\Lambda}$ are positive then r.w. is transient.

Proof. Let $\xi_{t}$ be the induced (ergodic) Markov chain, let $\eta(t, x)$ be mutually independent r.v., $x \in C^{\Lambda}, t=0,1, \ldots$, with values in $B^{\Lambda}$ so that

$$
P(\eta(t, x)=y)=\Sigma_{z} P \alpha \beta
$$

where $\alpha=x$ and the summation is over all $\beta$ such that $\beta=(y, z), y \in B^{\Lambda}, z \in C^{\Lambda}$. Then by ergodic theorem

$$
\frac{1}{n} \sum_{t=0}^{n-I} \eta\left(t, \xi_{t}\right) \rightarrow v \Lambda \text { a.s., } S_{n}=S_{0}+\sum_{t=0}^{n-I} \eta\left(t, \xi_{t}\right) .
$$

Then there exist sufficiently small $\varepsilon>0$ and $n_{0}>0, \delta>0$ such that for any ( $i \in \Lambda$ ). component $s_{n, i}$ of $s_{n}$

$$
s_{0}+\left(r_{i}^{\Lambda}-\varepsilon\right) n<s_{n, i}<s_{0}+\left(r_{i}^{\Lambda}+\varepsilon\right) n
$$

for all $n>n_{0}$ with probability not less than $\delta$. Taking $s_{0}$ sufficiently far from the boundary of $\Lambda$ we see that with positive probability we never reach this boundary and so by ergodic theorem we go to infinity. 


\subsection{Classification of paths}

We shall consider paths $\Gamma=\Gamma(t)$, i.e. continuous mappings $\Gamma:[0, T] \rightarrow \mathbb{R}_{+}^{N}, T$ can be equal to - , such that :

(i) $\Gamma(t)$ belongs to the union of ergodic faces except for a countable subset $\mathcal{J}=\mathcal{J}(\Gamma)$ of $[O, T]$;

(ii) for the points of the same interval belonging to $[O, T]-\mathcal{J}$ where the path runs through an ergodic face $B^{\Lambda} \Gamma(t)$ is linear with velocity $\frac{d \Gamma}{d t}=\mathrm{v}^{\Lambda}, \Gamma(t) \in B^{\Lambda}$.

Let us consider an ordered (in increasing order) sequence

$$
O \leqslant \ldots<t_{i}<\ldots t_{n}<\ldots
$$

of all the times $\Gamma(t)$ changes a face. It is countable and $\mathcal{J}$ belongs to this sequence. Note that sequence (1) can have accumulation points, e.g. it can approach towards and go away from anl-dimensional face rotating along 2-dimensional faces of $\mathbb{R}_{+}^{N}$ (see Fig.1, the one-dimensional face is perpendicular to the sheet of paper at the origin )

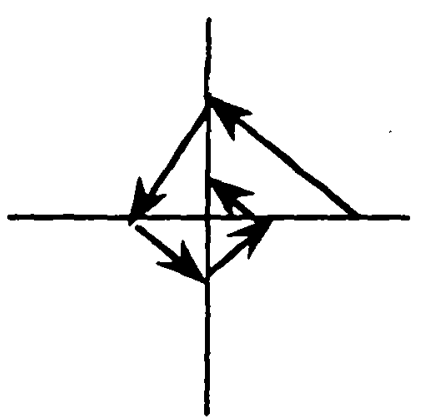

Fig.1a

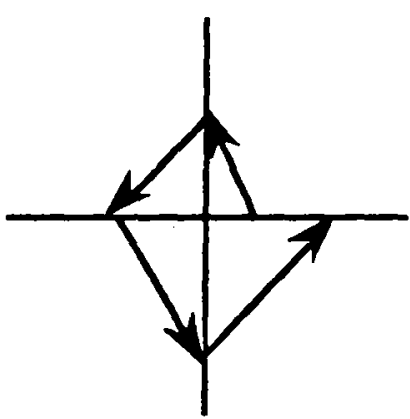

Fig.1b

So in general the sequence (1) is not isomorphic to the ordered set of natural numbers but in many examples it is (and so it is finite for finite $T$ ).

Together with the sequence (1) to any path $\Gamma$ is associated a denumerable ordered set of faces

$$
\Lambda_{1}(\Gamma), \ldots, \Lambda_{n}(\Gamma), \ldots
$$

which $\Gamma$ visits in the corresponding order.

The system of all different paths starting from $x \in \mathbb{R}_{+}^{N}$ is called an $x$-bundle (of paths) and is denoted by $V_{x}$, paths starting from $x$ are denoted by $\Gamma_{x}$. 


\section{Definition 1.3.1.}

Random walk is called regular if for any $x$ and $T$ there exists only a finite number $n\left(T, V_{x}\right)$ of paths $\Gamma_{x} \in V_{x}$ on the time interval $[0, T]$. It follows that the case on the Fig. $1 b$ is not regular as for any $T>0, x=0, n\left(T, V_{x}\right)$ is a continuum.

For regular random walk for any $\Lambda_{i}$ there exists the next face in the sequence (2) which is denoted by $\Lambda_{i+1}$.

A point $x$ is called stable if for any $\Gamma=\Gamma_{x} \in V_{x}$

$$
\operatorname{dim} \Lambda_{i}(\Gamma) \leqslant \operatorname{dim} \Lambda_{i+1}+1, i=1,2, \ldots
$$

whenever $\Lambda_{i+1}$ is the next face for $\Lambda_{i}$.

R.w. is strongly regular if for any stable $x, T<\infty$ and $\Gamma_{x}(t, t \in[0, T]$ the corresponding sequence (1) is finite. Strongly regular r.w. is regular and moreover it cannot have cases similar to Fig. 1a.

Further on we consider only strongly regular r.w. until otherwise stated.

Proposition 1.3.2. For any stable $x$, any $T, \varepsilon>0$ one can find $\delta>0$ such that for any $y$ with $y-x<\delta$, we have

1. $n\left(T, V_{x}\right)=n\left(T, V_{y}\right)$;

2. there is one-to-one correspondence $\phi: V_{x}(T) \rightarrow V_{y}(T)$ such that for any $\Gamma_{x} \in V_{x}(T)$ and $\Gamma_{y}=\phi\left(\Gamma_{x}\right) \in V_{y}(T)$ we have

$$
\left|\Gamma_{x}(t)-\Gamma_{y}(t)\right|<\varepsilon, t \in[0, T] .
$$

Proof follows by continuity if $\phi$ is chosen so that in the branch points $\Gamma_{x}$ and $\Gamma_{y}$ go to the same faces.

Lemma 1.3.3. There exists a subset $p 0 \subset$ of Lebesgue measure 0 (in fact it is a union of finite number of analytic hypersurfaces in $x^{2}$ ) such that for any regular random walk in 20 - 200 there exists at most denumerable number of hyperplanes in $R_{+}^{N}$ outside of which any point $x$ is stable.

Proof. In fact we shall construct a system of hyperplanes $B_{1}^{\Lambda}, \ldots, B_{k(\Lambda)}^{\Lambda}, k(\Lambda) \leqslant \infty$, for all ergodic $\Lambda$ with the following properties:

1. $B_{i}^{\Lambda} \subset B^{\Lambda}, B_{i}^{\Lambda} \neq B^{\Lambda}, i=1,2, \ldots, k(\Lambda)$, and $B_{i}^{\Lambda}$ are parallel to $v^{\Lambda}$;

2. any graph $\Gamma_{x}$ with $x \in B^{\Lambda}-\left(\cup_{i} B_{i}^{\Lambda}\right)$ is stable and otherwise it is not stable.

Proof. To construct such a system we use induction from lower dimensions. First for any $\Lambda, \Lambda_{1}$ such that $\Lambda \subset \bar{\Lambda}_{1}, \operatorname{dim} \Lambda \leqslant \operatorname{dim} \Lambda_{1}-2$, we consider the plane containing all the intervals $\left[x, x+\alpha v \Lambda_{1}\right], x \in \Lambda_{1}$, if $\exists \alpha>0$ such that $x+\alpha v \Lambda_{1} \in \Lambda$. 
If this plane does exist then it has dimension $\operatorname{dim} \Lambda+1$ and contains all path in $\Lambda_{I}$ which enter $\Lambda$ immediately after $\Lambda_{l}$.

All planes thus constructed we call ist generation planes.

We construct 2 nd generation planes in the following way : we take $\Lambda_{2} \neq \Lambda_{1}, \operatorname{dim} \Lambda_{2} \geq$ $\operatorname{dim} \Lambda_{1}$, and for any first generation plane in $\Lambda_{1}$ we consider all paths in $\Lambda_{2}$ which run along this plane immediately after $\Lambda_{2}$. The plane of minimal dimension containing all such paths is a 2 nd generation plane. Similarly we construct $3 r d$ generation planes etc. The planes of all generations comprise the desired system.

Now we find $\$ 00$ such that for any r.w. in $\$ 00$ any path $\Gamma_{x}$ with $x \in B^{\Lambda}$ $\left(\cup_{i} B_{i}^{\Lambda}\right)$ for an ergodic $\Lambda$ is stable.

Let us consider some path $\Gamma_{x}$ and in the corresponding sequence (1) only a subsequence of all ergodic chains

$$
\Lambda_{i_{1}}, \Lambda_{i_{2}}, \cdots
$$

Note that if $\operatorname{dim} \mathcal{f}_{n} \geq \operatorname{dim} \hat{i}_{n+1}$ for all $n$ then $\Gamma_{x}$ is stable. To deal with the cases when there exist $\hat{i}_{n}$ with $\operatorname{dim} \hat{i}_{n}<\operatorname{dim} \Lambda_{i_{n+1}}$ we define 200 in the following way : r.w. belongs to 200 iff there exists some $B_{i}^{\Lambda}$ containing some subface of the face $B^{A}$. Now let a sequence

$$
\Lambda_{1}, \Lambda_{2}, \ldots
$$

of ergodic chains exist such that the following conditions hold :

1. for any path $\Gamma_{x}:[0, \varepsilon] \rightarrow \Lambda_{1}$, there exists a path $\Gamma_{x}^{J}:[0, T] \rightarrow \mathrm{R}_{+}^{\mathrm{N}}$ coinciding with $\Gamma_{x}$ on $[0, \varepsilon]$ and having the sequence (3) as a sequence of ergodic faces visited by it ;

2. $\operatorname{dim} \Lambda_{2}>\operatorname{dim} \Lambda_{I}$;

3. for all $x \in \Lambda_{l}$ and all $k$ the path $I_{\mathrm{X}}^{l}$ belongs on $\Lambda_{k}$ to some of the planes $B_{i}^{\Lambda_{k}}$.

We see then that there exists $i, 2 \leqslant i \leqslant k$, such that $B_{i}^{\Lambda}$ contains some subface of the face $B^{\Lambda}$.

Note that we had not to take into account accumulation points in this proof .

Remark. The above construction vaguely resembles the construction of billiard dynamical systems [4].

\subsection{Associated dynamical systems}


Large time behaviour of a random walk depends only on its mactoscopic behaviour far from the origin. To decipher this vague statement we need some definitions.

Definition 1.4.1. R.w. is called deterministic if its second vector field has no branch points. Regular r.w. is called essentially deterministic if for any stable $x \quad \Gamma_{x}$ has no branch points.

Most of the difficulties appear already for essentially deterministic random walk (e.d.r.w.) and in this section we consider only this case defering scattering effects to the section 1.7 .

Let us consider e.d.r.w. in $\mathfrak{p}-\mathfrak{P}_{0}$ and let $T_{\mathrm{t}}^{l}$ be the dynamical system on $\mathrm{R}_{+}^{\mathrm{N}}$ defined by

$$
T_{t}^{l} x=\Gamma_{x}(t)
$$

for stable $x$, i.e. a point moves with velocity $v \Lambda$ along the ergodic face $\Lambda$. For non stable $\mathrm{x}$ we shall define $T_{t}^{l} x$ by continuity in every point where it is possible and in any suitable way otherwise.

Let us note that in earlier works we used exactly this dynamical system (which is a measurable flow) for classification of r.w.

Let us first note the following scaling property: for any $\alpha>0, t>0$

$$
\Gamma_{x}(t)=\alpha^{1} \Gamma_{\alpha x}(\alpha t)
$$

it holds not only for stable $x$ but for all $x$ if we take into account function $\phi$ defined as in the preceding section.

Let us consider convex polyedron $P^{N-1}=\left\{x \in \mathrm{R}^{N}: \Sigma_{i} x_{i}=I, x_{i} \geq 0\right\}$ and put $P \Lambda=$ $B^{\Lambda} \cap P^{N-1}$. We call $P^{\Lambda}$ ergodic if $\Lambda$ ergodic, etc. Most of the arguments below hold if $P^{N-1}$ is the (N-1)-dimensional sphere.

For each $x \in \mathbb{R}_{+}^{N}$ we denote $r(x) \subset \mathbb{R}_{+}^{N}$ the ray from the origin passing through $x$ and $e(x)$ its intersection with $P^{N-1}$.

Now we shall project paths $\Gamma_{x}(t)$ onto $\mathrm{pN}^{-1}$ by $\mathrm{e}\left(\Gamma_{x}(t)\right)$ and we shall need more information about these trajectories on $\mathrm{PN}^{\mathrm{N}-1}$.

Let us consider a face $B^{\Lambda} \subset R^{\operatorname{dim} \Lambda} \subset R^{N}$ and the plane $K^{\Lambda} \subset R^{\operatorname{dim} \Lambda}$ containing simplex $P \Lambda, \operatorname{dim} K^{\Lambda}=\operatorname{dim} \Lambda-1$. For any point $x \in K^{\Lambda}$ there is a unique decomposition

$$
v \Lambda=p^{\Lambda}(x)+r \Lambda(x)
$$


where the first vector lies in $K^{\Lambda}$ and the second is on the ray $O x$.

Trajectories of $e\left(\Gamma_{x}(t)\right)$ are straight lines but there are two cases:

Case 1. $\nu^{\Lambda}$ is parallel to $K^{\Lambda}$. Then $r \Lambda(x) \equiv 0$ and all trajectories are parallel and are intersections of the lines parallel to $M A$ with $P \Lambda$.

Case 2. $v \Lambda$ is not parallel to $K^{\Lambda}$, then there is a point $f^{\Lambda} \in K^{\Lambda}$ called a $\Lambda$-node such that trajectories of $e\left(\Gamma_{x}(t)\right)$ on $P \Lambda$ are the intersections of the rays in $K^{\Lambda}$, originating from this node, with $P \Lambda$. The node $f^{\Lambda}$ is the intersection of $K^{\Lambda}$ with the line $\{\alpha v \Lambda$ : $<\alpha<+\infty\}$. So the rays in question are intersections of $K^{\Lambda}$ with different two dimensional planes containing the line segment $\left(0 f^{A}\right)$.

Let us define the norm $n(x)$ on $R^{N}$ by

$$
n(x)=\frac{\|x\|}{\|e(x)\|}
$$

where $\|x\|=\left(\left(x^{1}\right)^{2}+\ldots+\left(x^{N}\right)^{2}\right)^{1 / 2}, x=\left(x^{1}, \ldots, x^{N}\right)$.

If we take the sphere $S^{N-1}$ instead of the simplex $P^{N-1}$ then $n(x)=\|x\|$.

We make a time change transformation by changing velocity $v(x) \rightarrow n(x) v(x)$

More exactly consider a new dynamical system $T_{t}^{2}$ defined by

$$
\frac{d\left(T_{t}^{2} x\right)}{d t}=n\left(T_{t}^{2} x\right) v\left(T_{t}^{2} x\right)
$$

(construction of the general time changing transformation see in [4]).

Definition 1.4.2. Our basic dynamical system on $P^{N-1}$ is defined by projecting $\tau_{t}$ onto $\mathrm{PN}-1$

$$
T_{1} \omega=e\left(T_{t}^{2} x\right), \omega=e(x) \in p^{N-1} .
$$

and we easily see that (2) is consistent, i.e. does not depend on $n(x)$. In fact, moreover

$$
\begin{aligned}
& \lim _{\varepsilon \rightarrow 0} \frac{T_{\varepsilon}(\omega-\omega}{\varepsilon}=\lim _{\varepsilon \rightarrow 0} \frac{1}{\varepsilon}\left(e\left(T_{\varepsilon}^{2} x\right)-\omega\right)= \\
& =\lim _{\varepsilon \rightarrow 0} \frac{1}{\varepsilon}(e(x+\varepsilon n(x)(p(x)+r(x)))-\omega)=\lim _{\varepsilon \rightarrow 0} \frac{1}{\varepsilon}(e(n(x)(\omega+\varepsilon(p(\omega)+r(\omega))))-\omega)= \\
& =\lim _{\varepsilon \rightarrow 0} \frac{1}{\varepsilon}\left(e\left(\omega+\varepsilon\left(p(\omega)+r(\omega+e p(\omega))+O\left(\varepsilon^{2}\right)\right)-\omega\right)=\rho(\omega)\right.
\end{aligned}
$$

Again for nonstable $\omega$ we define $T_{\uparrow} \omega$ by continuity whenever it is possible. 
It is useful also to consider discrete time versions (Poincaré maps) of such dynamical system. Let us consider a closed subset $\Omega \subset \mathrm{pN}^{-1}$ with the following properties :

(i) for any stable $\omega$

$0<\pi(\omega ; \Omega)=\inf \{s>0: T, \omega \in \Omega\}<\infty$;

(ii) subset of stable points is open and dense in $\Omega$.

We define the transformation $U \equiv U_{\Omega}: \Omega \rightarrow \Omega$ by

$$
U \omega \equiv T_{t(\omega, \Omega)} \omega \text {. }
$$

Then $T_{t}$ is a kind of special flow over $U$, see $[4]$.

There exists $s(t, \omega)$ such that

$$
T_{s(t, \omega) \omega}=e\left(\Gamma_{\omega}(0)\right)=e\left(\Gamma_{r \omega}(r 0)\right)
$$

by scaling property for any $r>0$. As $s(t ; \omega)$ is strictly increasing we can consider the inverse function $\pi s, \dot{\omega})$ so that $s(\pi, \omega)=s$.

The constructed dynamical system is related to r.w. via the law of large numbers. We recall one result from [ 2 ].

Let us fix some stable $\omega \in P^{N-1}$ and choose a sequence of points $x(n) \in Z_{+}^{N}, n \rightarrow \infty$, so that

$$
\|x(n)-n \omega\|=o(n)
$$

and let $\xi_{x(n)}(\tau)$ be the random walk starting from $x(n), \tau=0,1, \ldots$

Definition 1.4.3. We say that regular e.d.r.w. is inductively stable (or inductive) if for any $\varepsilon>0,1>0$, stable $\omega$

$$
P\left(\left|\xi_{x(n)}(t n)-\Gamma_{a n}(t n)\right|>\varepsilon n\right) \rightarrow 0, n \rightarrow \infty
$$

We see that stability of the initial point of a path plays a crucial role because we have an exactly defined drift in all the points of the path. In other words regular essentially deterministic inductive r.w. and stable $\omega$ for any fixed $t>0$ random variable $e\left(\xi_{x, n}(t n)\right)$ tends to a constant vector $T_{*(1, \omega)} \omega$ in probability if $n \rightarrow \infty$.

If $\Gamma_{\omega}(s)$ lies on the same ergodic face $\Lambda$ for all $0 \leq s \leq t$ then (5) holds under some mild assumptions about the induced chain $\mathfrak{L}_{\Lambda}$.

Problem 1.4.1. Is this true for any random walk and any $\Lambda$ ? Examples when it is not the case are not known.

Theorem 1.4.2. If $N \leqslant 4$ then regular e.d.r.w. is always inductive with possible exceptions of zero measure in $\mathrm{s}$. 
The proof is implicit in $[2,10]$.

We discuss below a lot of examples and it is very important for us that corresponding r.w. be inductive. We claim that it is for a positive measure in parameter space. The general principle is the following : if all induced chains of a r.w. have sufficiently nice Lyapounov functions then this r.w. is inductive. Unfortunately I do not know sufficiently general nicely formulated result which could not be immediately generalised. Example of such a result is

Proposition 1.4.3. If for all ergodic and nonergodic faces the conditions of Lemma 2.3 in [2] are satisfied then the chain is inductive.

\subsection{Multiplicative suspensions over dynamical systems}

Our philosophy here is to consider r.w. as a scaled random perturbation of the dynamical system $T_{t}$ constructed in section 1.4. This enables us to get new results and comprehend previous ones more appropriately. However complete solution of the problem is not possible. The difficulties are the same as for general ergodic theory (we shall see later that sufficiently general dynamical systems arise for r.w. in $\mathbf{Z}_{+}^{N}$ ). So it is quite reasonable to visualise the problem from a more general point of view. One easily sees two levels of approximation to our problem.

1 level. It is the dynamical system $T_{t}^{l}$ but with an additional probabilistic structure.To introduce it we give the new definition.

Let $M$ be a compact metric space, $B$ its Borel $\sigma$-algebra, $T: M \rightarrow M$ - a transformation measurable w.r.t. 3 . Probability measure $\mu$ on $M$ is called invariant if $\mu\left(T^{-1} A\right)=\mu(A)$ for any $A \in \mathbb{B}$. Let $\mathfrak{M}(M, T)$ be a set of all such invariant measures. One of dynamical systems we shall consider on $M=P^{N-1}$ is $T=T_{t}$ with some fixed $t$ or on $M=\Omega$ with $T=U$. We could equally consider the flow $T_{t}$ - a reader can make necessary reformulations..

Definition 1.5.1. In the abstract setting let be given an array $(M, T, \mu), \mu \in \mathbb{M}(M, T)$, and a positive function $f(\omega)$ on $M$. We always assume that for some positive constants $a_{1}$, $a_{2}, a_{1}<f(\omega)<a_{2}$. A multiplicative suspension over $(M, T, \mu)$ is the dynamical system $T^{\prime}$ on $M \times R_{+}$defined by

$$
T^{\prime}(\omega, r)=(T \omega, f(\omega) r) .
$$

Remark. Multiplicative suspension has nothing to do with the standard term "suspension" in dynamical system theory.

In our case 


$$
f(\omega)=\frac{\left\|\Gamma_{\omega}(t)\right\|}{\|\omega\|}
$$

with fixed $t$ when we consider $\left(P^{N-1}, T_{t}\right)$ and with $t=\tau(\omega ; \Omega)$ when $T=U$ is a dynamical system on $\Omega$. So

$$
\left(T^{\prime}\right)^{n}(\omega, r)=\left(T^{n} \omega, \chi_{n} r\right)
$$

where

$$
\chi_{n}=\chi_{n}(\omega)=\prod_{i=0}^{n-1} f\left(T^{i} \omega\right)
$$

Let also be given a nonnegative everywhere finite measurable function $t(\omega, r)$ on $M \times R_{+}$ which is called local time. It is assumed to have the following property :

$C_{1} r f(\omega) \leqslant t(\omega, r) \leqslant C_{2} r f(\omega)$ for some constants $C_{1}, C_{2}>0$.

Random variables

$$
t_{n}(\omega ; r)=\sum_{i=0}^{n-1} t\left(T^{i} \omega, \chi_{i}\right)
$$

are called proper times (starting from the point $(\omega, r)$ )

We know that for large $n$

$$
\frac{\left\|\xi_{n \omega}(n t)\right\|}{\left\|\xi_{n \omega}(0)\right\|} \approx f(\omega) \equiv \frac{\left\|\Gamma_{\omega}(t)\right\|}{\left\|\Gamma_{a}(0)\right\|} .
$$

So we could expect that the behaviour of the modulus of the random walk is defined by the product

$$
f(\omega) f(T \omega) . . . f\left(T^{m-1} \omega\right)
$$

or by

$$
\frac{l}{m} \log \left(f(\omega) f(T \omega) . . . f\left(T^{m-1} \omega\right)\right)
$$

if its behaviour for $m \rightarrow \infty$ is suffuciently sharp.This heuristical hints lead us to the following exact definitions.

Definition 1.5.2. We call a multiplicative suspension

(i) recurrent if for all $r_{0}>0$ a sequence of random variables

$$
\chi_{n}=\chi_{n}(\omega)=\prod_{i=0}^{n-1} f\left(T^{i} \omega\right)
$$

reaches $r_{0}$ - neighbourhood of $0 \mu$-a.s., that is

$$
\tau=\tau\left(\omega ; r_{0}\right)=\min \left\{n: \chi_{n}(\omega)<\text { ro }\right\}<\text { - a.s }
$$

It is equivalent of course to require that $t_{\tau}$ is finite a.s. 
(ii)transient if it is not recurrent;

(iii) positive recurrent if

$$
E_{\mu} t_{\tau}<\infty
$$

(iv) null recurrent if it is recurrent but not positive recurrent.

By ergodic theorem the following limit exists almost everywhere

$$
\lim _{n \rightarrow \infty} \frac{1}{n} \log \prod_{i=0}^{n-1} f\left(T^{i} \omega\right)=L(\omega)
$$

and

$$
L(\omega)=L(T \omega)
$$

If $T$ is ergodic then

$$
L(\omega)=L \equiv \int_{\Omega} \log f(\omega) d \mu(\omega) .
$$

For ergodic $T$ let us assume that the following limit also exists

$$
M=\lim _{n \rightarrow \infty} \frac{1}{n} \log E \prod_{i=0}^{n-1} f_{i}
$$

also exists.

Remark 1.5.1. By Iensen's inequality

$$
L \leqslant M
$$

$L$ is a trivial case of a Lyapounov exponent, $M$ is a free energy. It is a difficult problem when $M$ does exists.

In many cases $L=M$, the simplest case when it is not the case appears in [12] for random walks which are not essentially deterministic (see section 2.2). See section 4.2 for e.d.r.w. when $M \neq L$.

Lemma 1.5.2. Let $T$ be ergodic and

$$
L \neq O, M \neq O
$$

Then the multiplicative suspension is

(i) recurrent iff $L<0$;

(ii) transient iff $L>0$;

(iii) positive recurrent iff $M<0$;

(iv) null recurrent iff $L<0, M>0$.

Proof. If $L<O$ then by ergodic theorem $\chi_{n}(\omega) \rightarrow 0$ a.s. So multiplicative suspension is recurrent. If $L>0$ the $\chi_{n}(\omega) \rightarrow \infty$ a.s. and so (due to the boundedness of $f$ ) there exist $A \subset \Omega, \mu(A)>0$, and $r_{0}>0$ such that for all $\omega \in A, \chi_{n}(\omega)>r_{0}$ for all $n$. 
If $M<O$ then

$$
\left.E \pi T \omega, \chi_{n}(\omega)\right) \leqslant C_{2} E \chi_{n}(\omega) f(\omega) \leqslant C e^{-m}, \gamma<0 .
$$

So even

$$
\left.\sum_{n=1}^{\infty} t^{n} \omega, x_{n}(\omega)\right)<\infty .
$$

If $M>0$ then for $\varepsilon>0$ there exists $n_{0}=n_{0}(\varepsilon)$ such that

$A_{n}=\left\{\omega: \chi_{n}>e^{(M-\varepsilon) n}\right\}$

has positive measure $\mu$ for all $n>n_{0}$ and moreover

$$
\int_{A_{n}} t_{n}(\omega ; 1) \mu(\omega)>e^{(M-\varepsilon) n} .
$$

Fix $\varepsilon>0$ sufficiently small and then take ro sufficiently small. We want to prove that $E_{\mu} t_{\tau}=\infty$, for $\tau=\tau\left(\omega, r_{0}\right)$

For fixed $n, \omega \in A_{n}$ let us choose all pairs $D \leqslant i d(\omega)<i_{n}(\omega) \leqslant n$ such that $\chi_{i_{0}}(\omega) \leqslant D, \chi(\omega)>D, i_{0}<i<i_{1}, i_{1}=\min \left\{n, j: j>i_{0}, \chi(\omega)<D\right\}$.

We choose $D>0$ and note that $i d(\omega)$ can be not unique. Let us put

$$
B(n)=\left\{T \omega: \omega \in A_{n}, j=i \delta(\omega)\right\}
$$

Then

$$
\int_{B(n)} t_{\tau}(\omega ; 1) d \mu(\omega)>\int_{A_{n}} t_{n}(\omega ; 1) d \mu(\omega)-C D n
$$

for some constant $C>0$. Then with $n \rightarrow \infty, E_{\mu} t_{\tau}=\infty$ follows.

Unfortunately we do not know which invariant measure $\mu \in \mathcal{M}_{(M, T)}$ defines the behaviour of our r.w. This is in fact one of the main difficulties to be discussed below. Lemma 1.5.2 holds also for the case when $T_{t}$ is a Markov process with an invariant measure $\mu$. Much less is known for the case when either $L$ or $M$ is equal to 0 (see Appendix).

Level 2. Generally one cannot hope characterize r.w. in terms of the random suspension of the dynamical system $(M, T)$ because it can have continuum invariant measures with the same support and it is not clear which of them is responsible for the calculation of constants $M$ and $L$. The choice of the right measure is provided by a small random perturbation.

We shall define now a scaled random suspension. It is in fact exactly r.w. but without taking into account the arithmetical nature of the lattice $Z_{+}^{N}$. All known examples show that neither arithmetical properties of the lattice nor an exact form of the random 
perturbation (see below) do not play role and one can suggest the universality hypothesis which says that ergodicity, null recurrence or transience of a r.w. are the same as ones for the scaled random suspension defined below.

Problem 1.5.1. Find examples when universality hypothesis does not hold.

We shall give definitions only for discrete time dynamical systems $T_{t}$ or $U$.

Definition 1.5.2. Random perturbation of the dynamical system $(M, T, \mu)$ is a Markov process with transition measures $Q\left(\omega, d \omega^{\prime} \varepsilon\right)$ which depend on parameter $\varepsilon>0$ so that $\rho\left(\omega, \delta \omega^{\prime}, \varepsilon\right)$ tends weakly to $\delta$ measure in the point $T \omega, \omega \in M$, when $\varepsilon \rightarrow 0$. Scaled random suspension (perturbation) of the dynamical system $(M, T, \mu)$ is a Markov process on $M \times R_{+}$defined by transition measures

$\left.P\left((\omega, r),\left(d \omega^{\prime}, d r^{\prime}\right)\right)=Q\left(\omega, d \omega^{\prime} ; r^{-1 / 2}\right) \& r^{\prime}-r f(\omega)\right) d r^{\prime}$

for some random perturbation $Q$.

See $[21,22,44]$ for deeper discussions on random perturbations.

1.6. About scaled random perturbations.

Multiplicative matrix functionals over dynamical systems is a well developped branch of mathematics since the work by V.I.Oseledec [26] , see [23].

Has'minskii and others [ $16-18]$ studied the stability of linear Ito equations

$$
d x=\left(A+\frac{1}{2} \sum_{i=1}^{m} B_{i}^{2}\right) x d t+\sum_{i=1}^{m} B_{i} x d W_{i}, \quad x(0)=x_{0}
$$

where $A, B_{i}-(N \times N)$-matrices, $W_{i}$ - mutually independent standard Wiener processes. The crucial property here, allowing to calculate the top Lyapounov exponent, is that the projection

$$
s(t)=\frac{x(t)}{\|x(t)\|}
$$

of the equation (1) onto the unit sphere is a Markov process. The invariant measure of this process admits sufficient control and $x(t)$ is just a functional over this process. Moreover no feedback appears : $\|x(t)\|$ does not influence $s(t)$. It is easy to see that the reason is that the equation (1) is scale invariant, i.e. drift and diffusion coefficients are linear in $\|x(t)\|$. In our case they are constant (do not depend on $\|x(t)\|$ ) and so a scaled random suspension arises the invariant measures of which are much harder to control. There is mutual interaction : scale influences the dynamical system. 
Related to our problems is the following solvable problem conceming equations of type (1).

Problem 1.6.1. Let (1) be only piecewise linear, i.e. matrices $A$ and $B_{i}$ are constant in some cones. Classification of such systems allows to make comparisons of its recurrence and transience with our situation for the case when $P(N ; k)$ ( see below in section 3.4 ) is a sphere.

\subsection{Scattering with finite exit boundary.}

We consider here strongly regular r.w. which are not essentially deterministic.

For a nonergodic face $\Lambda$ let $S^{+}(\Lambda)\left(S^{-}(\Lambda)\right)$ be the set of all ingoing (outgoing) faces for $\Lambda$. It is useful to describe a structure of $s(\Lambda)$.

Proposition 1.7.1. If $\Lambda_{1}, \Lambda_{2} \in S^{-}(\Lambda)$ then neither $\Lambda_{1} \subset \Lambda_{2}$ nor $\Lambda_{1} \supset \Lambda_{2}$.

Proof. If $\Lambda_{2} \in S^{-}(\Lambda)$ then $\checkmark^{\Lambda_{2}}$ has all components $\nabla_{i}, i \in \Lambda_{2}-\Lambda$, positive. Then $\Lambda_{1}, \Lambda_{1} \subset \Lambda_{2}$, is not ergodic. See proposition 1.2.3.

Let be given a stable $\omega \in P^{\Lambda^{+}}, \Lambda^{+} \in S^{+}(\Lambda)$, and $\Gamma_{\omega}(t) \in V_{\omega}, t \in[0, T]$, such that there exists $t_{1} \in[0, T]$ with the properties: $\Gamma_{\omega}\left(t_{1}\right) \in \Lambda, \Gamma_{\omega}(t) \in \Lambda^{+}, t \in\left[0, t_{1}\right)$, $\Gamma(t) \in \Lambda^{-},, t \in(t,, T)$. As $\omega$ is stable

$$
\operatorname{dim} \Lambda^{+}=\operatorname{dim} \Lambda+1 \text {. }
$$

Definition 1.7.1. Assume that all $p_{\alpha \beta}$ are different from zero for $\|\alpha-\beta\| \leqslant 1$. We say that a strongly regular r.w. is inductive if there exist scattering probabilities $p^{\Lambda}\left(\Lambda^{+}, \Lambda^{-}\right)>0, \quad \sum p^{\Lambda}\left(\Lambda^{+}, \Lambda^{-}\right)=1$, such that for any sufficiently small $\delta>0$ any $\Lambda^{-} \in S^{-}(\Lambda)$

$\omega, T, \Gamma_{\omega}(n)$, satisfying the above conditions, in notations of Definition 1.4.1

$$
\left|P\left(\left|\xi_{x(n)}(T n)-\Gamma_{a m}(T n)\right|<\delta n\right)-p^{\Lambda}\left(\Lambda^{+}, \Lambda^{-}\right)\right| \rightarrow 0, n \rightarrow \infty
$$

Theorem 1.7.2. Let us consider strongly regular inductive r.w. and assume that all $p_{\alpha \beta}$ are different from zero for $\|\alpha-\beta\| \leqslant 1$. Let $\Gamma_{\alpha} \gamma(0)$ be any path with $\omega \in p^{\Lambda^{+}}$, let $\Lambda_{i}$ be all nonergodic faces and $\Lambda_{i}^{+}, \Lambda_{i}^{-}$be all corresponding pairs of ingoing and outgoing faces which $\Gamma_{\omega}(t)$ visits. Then for any $T>0$

$$
\mid P\left(\left|\xi_{x(n)}(T n)-\Gamma_{a n}(T n)\right|<\delta n\right)-\prod_{i} p^{\Lambda_{i}\left(\Lambda_{i}^{+}, \Lambda_{i}^{-}\right) \mid \rightarrow 0, n \rightarrow \infty}
$$


Corollary 1.7.4. Under the conditions of the preceding theorem

${ }^{\min } \Gamma_{\omega \in V_{\omega}} \rho\left(\xi_{x(n)}(T n), \Gamma_{a n}(T n)\right) \rightarrow 0$

in probability when $n \rightarrow \infty$.

For this case the same remarks as for inductive e.d.r.w. can be made. In small dimensions see the proof of inductiveness in $[10,12]$.

In fact in definition 1.7.1 it is claimed that the exit boundary for the induced Markov chain is finite.

In this case we define instead of the associated dynamical system $T_{t}^{\prime}$ the Markov process which is "random" only in some discrete moments when it hits a nonergodic face where it chooses along which outgoing faces it should go. It makes this choice instantaneously with the corresponding scattering probabilities. It moves along ergodic $P \Lambda$ as it was described below. In the same way it is possible to define restrictions $(\Omega, U)$ of this process. E.g. in dimension $4 \Omega$ can be always chosen finite.

\section{ACYCLICITY AND SMALL DIMENSIONS}

\subsection{Dimensions 1, 2, 3 and acyclic random walks.}

All cases for dimensions 1, 2, 3 can be described by the following theorem.

Theorem 2.1.1. For $N=1,2,3$ under assumptions $O_{1}, O_{2}$ r.w. is ergodic iff every path $\Gamma_{x}(t)$ for stable $x$ reaches the origin. If at least one such path goes to infinity then the r.w. is transient.

It is very instructive to look at the earlier results from the novel point of view. It appears that the corresponding dynamical systems on $P^{N-1}$ are very simple. On the language of associated dynamical system theorem 2.1.1 can be restated as follows.

Theorem 2.1.1'. For $N=1,2,3$ under assumptions $\mathrm{O}_{1}, \mathrm{O}_{2}$ r.w. is ergodic iff invariant point measures in all stable fixed (or periodic) points have $L<0$. If for at least one $L>0$ then it is transient.

Now we shall consuder it in more detail.

Dimension 1. Here the dynamical system $T_{t}$ is trivial, $P^{0}$ is a one-point set $\{1\} \in$ $\mathrm{R}_{+}^{1}, v^{\{1\}}$ is just the mean jump $M_{\{1\}}$,so the second vector field coincides with the first. It is a classical result [7] that if $M_{\{1\}}<0$ (so any path $\Gamma_{x}$ goes straightly to 0 ) then r.w. is ergodic, and $M_{\{1\}}>0$ ( so any path $\Gamma_{x}$ goes straightly to $*$ ) implies transience. 
Dimension 2. This case was completely solved in [8] under assumptions $\mathrm{O}_{1}, \mathrm{O}_{2}$. Only the first vector field was used in [8]. Connection with the second vector field was described in [2].

Here $P^{1}$ is the interval $\{(x, y): x+y=1, x, y \geq 0\}$ with two boundary points $(0,1)$ and $(1,0)$. There can be four cases (see Fig. 1) for $T_{t}$

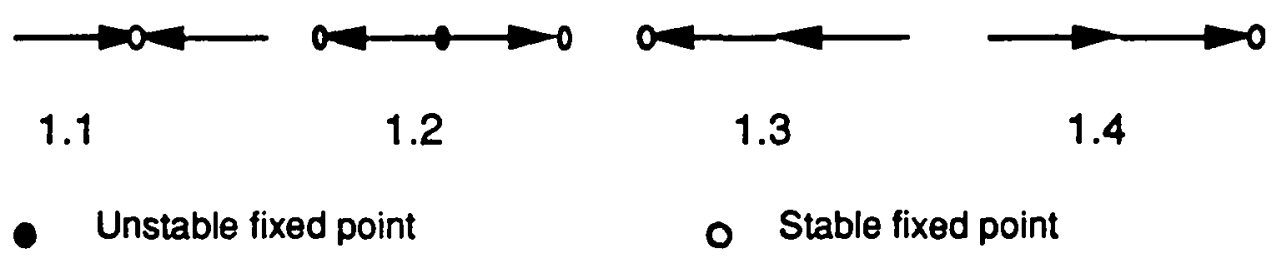

Fig. 2

1.1. The node $f^{\Lambda}$ lies inside $P^{l}$ and is attractive. This is equivalent to the statement that both components of $w_{\{1,2\}}$ are positive. Both one-dimensional faces are nonergodic and any path goes to $\infty$. R.w. is transient. See proposition 1.2.3.

1.2. The node $f^{\wedge}$ lies inside $P^{l}$ and is repulsive. This means that both components of $v\{1,2\}$ are negative. Both one-dimensional faces are ergodic. If

$$
v\{1\}<0, v\{2\}<0
$$

then all the paths go to 0 and r.w. is ergodic. Condition (1) can be written in terms of the first vector field as the induced chains are one dimensional r.w. (see $[2,12]$ ).

If at least one of $v^{\{i\}}$ is positive then there are stable paths going to $\infty$ and r.w. is transient.

1.3. The node $f^{\Lambda}$ lies from the left of the interval $((0,1),(1,0))$. So $y$-axis is ergodic and r.w. is transient if $v^{\{2\}}>0$ and ergodic if $v\{2\}<0$.

\subsection{Symmetric to 1.3 .}

All these cases can be summarised in the following theorem.

Theorem 2.1.2. R.w. is transient iff there is at least one attractive fixed point of the dynamical system in $P^{I}$ among $f^{\Lambda},(0,1),(1,0)$ and in this point the corresponding vector $v$ has all its nonzero components positive. In the other words for at least one of the invariant measures we must have $L>0$.

Here $L=M$ and e.g. for $(0,1)$ they coincide with $v\{2\}$. Signs of $v \Lambda$ for onedimensional $\Lambda$ can be calculated in terms of the first vector field (see $[2,12]$ ).

Dimension 3. Necessary and sufficient conditions were obtained in $[9,2]$ also under assumptions $\mathrm{O}_{1}, \mathrm{O}_{2}$ (for the case 2.7 we need one more assumption). 
Here $P^{2}$ is the (closed) two dimensional simplex.All typical cases are clear from the Fig. 2 .

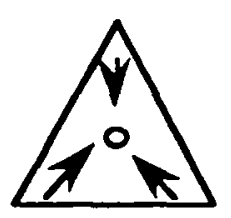

1

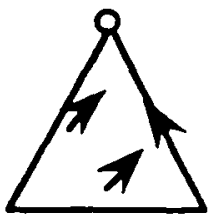

5.

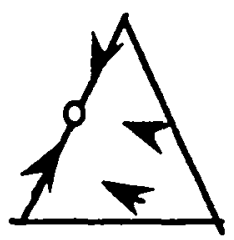

8

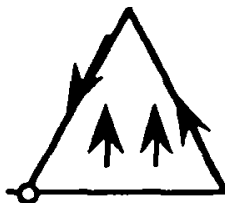

2

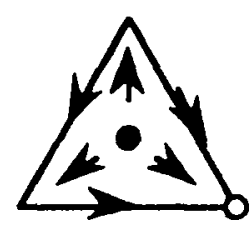

6

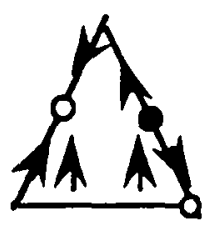

9

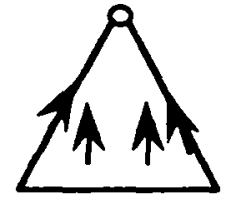

3

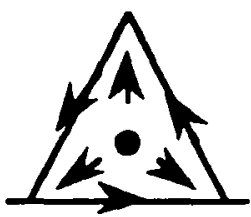

7

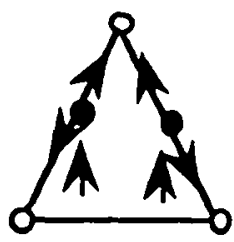

10

Fig. 3

2.1. The node $f^{\wedge}$ lies inside $P^{2}$ and is attractive. This is equivalent to the statement that both components of $v\{1,2,3\}$ are positive. All two-dimensional faces are nonergodic and any path goes to $\infty$. R.w. is transient.

2.2-4. There are two ergodic two-dimensional faces and one $(2.2,2.3)$ or two (2.4) ergodic one-dimensional faces which correpond to stable fixed points on Fig.2. R.w. is ergodic iff all vectors ${ }^{\Lambda}$ corresponding to ergodic one-dimensional faces are negative. Here a sign of this $w \Lambda$ has no simple form but in some cases (components of the one step jumps of the r.w. are bounded by 1 ) can be expressed in terms of elliptic integrals (see $[34,35])$. This was the first example when ergodicity conditions are not of elementary form.

2.5. One two-dimensional face is ergodic and one one-dimensional face $\Lambda$ is ergodic. R.w. is ergodic iff the sign of $w \Lambda$ is negative.

2.6. All two dimensional faces are ergodic, only one one-dimensional face $\Lambda$ is ergodic. R.w. is ergodic iff the sign of $v \Lambda$ is negative. 
2.7. All two dimensional faces are ergodic but there is no ergodic one-dimensional faces. Here it is convenient to choose a dynamical system $(\Omega, U)$ as follows. We take $\Omega$ as a three point set $\left\{\omega_{1}, \omega_{2}, \omega_{3}\right\}$ - all vertices of $P^{\prime} . U$ acts cyclically $\omega_{i} \rightarrow \omega_{i+1}(\bmod 3)$. Let $\omega_{i}$ correspond to the one-dimensional face $x_{i}>0$. Then

$$
f\left(\omega_{i}\right)=\left|\frac{v_{i+1}^{\{i, i+1\}}}{v_{i}^{\{i, i+1\}}}\right|,
$$

that is $\operatorname{tg} \phi$ where $\phi$ is the angle between the vector $v\{i, i+1\}$ and the face $x^{i}>0$.

R.w. is ergodic if

$$
L \equiv \frac{1}{3} f\left(\omega_{1}\right) f\left(\omega_{2}\right) f\left(\omega_{3}\right)<0
$$

and transient if $L>0$.

Here $L=M$. We do not consider the case $L=0$.

2.8 - 2.10. There are a lot of cases where fixed points of $T_{\imath}$ lie inside one-dimensional simplexes.

The following proposition comprises all cases except for the case 2.7.

Proposition 2.1.3. If there is at least one stable point inside two or one-dimensional simplex then r.w. is transient. Otherwise it is transient iff there exists stable vertex of $p^{2}$ with positive $\nu^{\Lambda}$ for corresponding one-dimensional face $\Lambda$.

It is a part of the theorem 2.1.1'.

All these cases (except 2.7) and also many examples in higher dimensions belong to the following class of r.w..

Definition 2.1.1. A path $\Gamma_{x}, x \in \mathbb{R}_{+}^{N}$, is called acyclic if it visits any face not more than once, i.e. if the sequence $\Lambda_{i}\left(\Gamma_{x}\right)$ is finite and all $\Lambda_{i}\left(\Gamma_{x}\right)$ are mutually different. R.w. is called acyclic if all paths are acyclic.

Definition 2.1.1. For any $x$ (not necessary stable) let $V_{x}(v)$ be the set of limiting points of $\Gamma_{x_{n}}$ ( $)$ for all sequences of stable $x_{n} \rightarrow x$. We call r.w. completely inductive if for any $\omega$, any $T$ and $n \rightarrow \infty$

$$
\text { inf } \rho\left(\xi_{x(n)}(T n), V_{x(n)}\left(T_{n}\right)\right) \rightarrow 0
$$

Problem 2.1.1. Acyclic r.w. is completely unductive. It is ergodic iff for any stable $x$ $\Gamma_{x}(t)$ reaches to the origin.

It is not always easy to verify that a given r.w. is acyclic because of the nontriviality of calculation of the second vector field. For instance for Jackson networks where simple Lyapounov function is now constructed [29] but still exists

Problem 2.1.2. Prove that ergodic Jackson network is acyclic. 


\subsection{Scattering in 2-dimensional complexes.}

Let us consider as a typical example the following r.w. in $\mathbf{Z}_{+}^{N}$. All $M_{\Lambda}, \operatorname{dim} \Lambda \geqslant 3$, are assumed to have all their components negative. This implies that all two-dimensional faces are ergodic and that the r.w. is inductive. Let $\Omega$ be the set of all two-dimensional faces of $\mathbf{Z}_{+}^{N}$ and $S^{+}(\Lambda)\left(S^{-}(\Lambda)\right)$ a set of ingoing(outgoing) two-dimensional faces of a one-dimensional face $\Lambda$. We assume that all $\boldsymbol{v}, \operatorname{dim} \Lambda=1$, have one component negative and the other positive. It follows that all $S^{+}(\Lambda)$ for different $\Lambda$ are mutually disjoint (the same is for $S^{-}(\Lambda)$ ).

Lemma 2.2.1. Under these conditions r.w. is inductive.

So for any $\Lambda^{+} \in S^{+}(\Lambda), \Lambda^{-} \in S^{-}(\Lambda)$ the scattering probabilities $p\left(\Lambda^{+}, \Lambda^{-}\right)$exist.

Definition 2.2.1. The associated (with the r.w. in question) Markov chain $(\Omega, T)$ with the state space $\Omega$ and stochastic transition matrix $T=\left\|p\left(\Lambda^{+}, \Lambda^{-}\right)\right\|$.

Assumption $\mathrm{O}_{3}$. Let us assume that $(\Omega, T)$ is irreducible and aperiodic.

Then let us put

$$
f(\Lambda)=\operatorname{tg} \phi_{\Lambda}
$$

where $\phi_{\Lambda}$ is the angle between $v^{\Lambda}$ and the negative direction of the one-dimensional axis from which ${ }^{\wedge}$ goes away.

Lemma 2.2.2. The following limits exist

$$
\begin{aligned}
& L=\lim _{n \rightarrow \infty} \frac{1}{n} E \log \prod_{i=0}^{n-1} f\left(\Lambda_{i}\right) \\
& M=\lim _{n \rightarrow-\infty} \frac{1}{n} \log E \prod_{i=0}^{n-1} f\left(\Lambda_{i}\right)
\end{aligned}
$$

where $\Lambda_{i}$ is the associated Markov chain. They are equal to

$$
L=\Sigma_{\Lambda} \pi(\Lambda) \pi(\Lambda), \quad M=\log \lambda_{1}
$$

where $\pi(\Lambda)$ are the stationary probabilities for the associated (finite) Markov chain and $\lambda_{1}$ is the maximal eigenvalue of $n \times n-$ matrix, $n=|\Omega|$,

$$
A\left(\Lambda^{+}, \Lambda^{-}\right)=\rho\left(\Lambda^{+}, \Lambda^{-}\right) \sqrt{\kappa\left(\Lambda^{+}\right) \pi\left(\Lambda^{-}\right)}
$$

Assumption $\mathrm{O}_{4}, L \neq O, M \neq O$ 
Theorem 2.2.3. Under the above conditions r.w. is

(i) recurrent iff $L<0$;

(ii) transient iff $L>0$;

(iii) positive recurrent iff $M<0$;

(iv) null recurrent iff $L<0, M>0$.

Scattering probabilities can be explicitly calculated in some cases (see [12]). Complete classification see in [12].

We want to emphasize that inessential states of the associated Markov chain can play crucial role in the ergodicity of the r.w. and so stationary distribution of the associated Markov chain does not always characterize the ergodicity of r.w. Nevertheless the stationary distribution of the associated Markov chain always characterize recurrence and (and transience).

\subsection{Dimension 4.}

In dimension 4 the simplex $P^{3}$ is the tetrahedron - one of the famous Platonian convex bodies. In this case it is the surface of this tetrahedron where the scenario is being played. For the dynamical system on 2-dimensional sphere Poincare-Bendixson theory has to be used. We do not give the complete description of results (see [10]) but only some typical examples (see Fig. 4). 


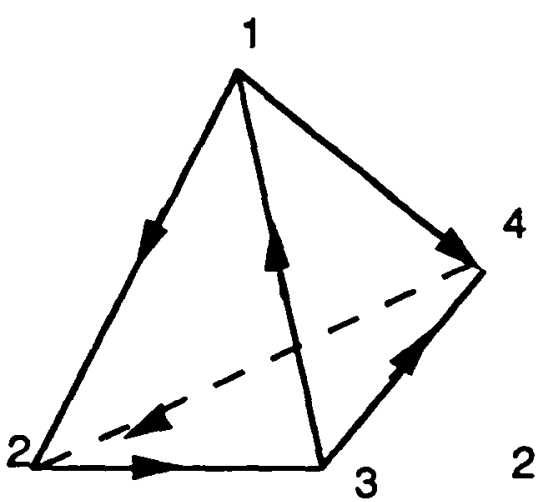

1

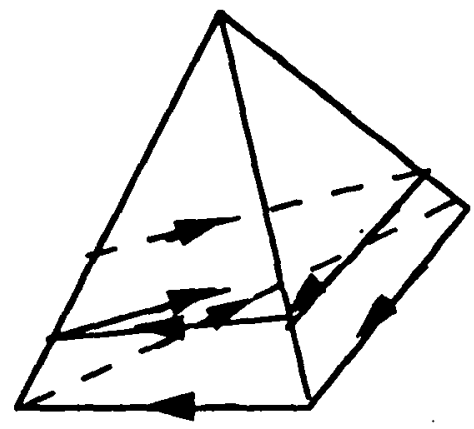

4

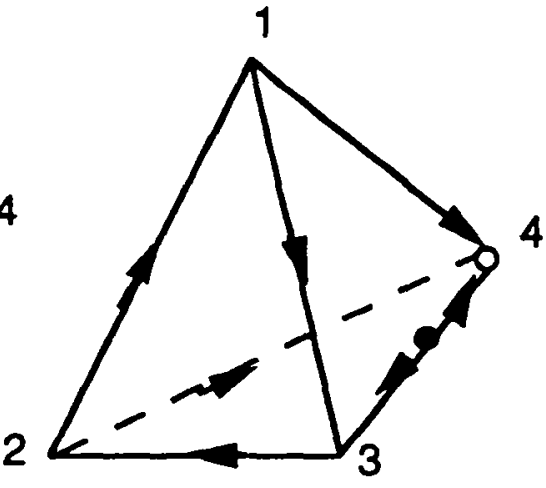

2

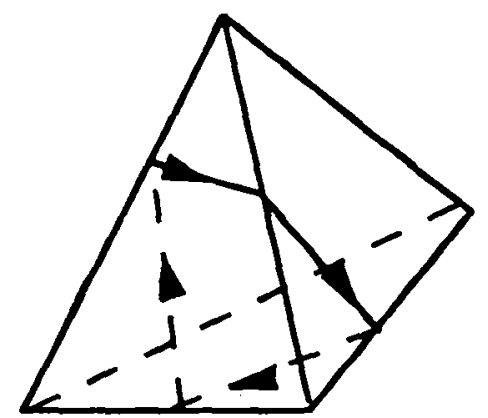

5
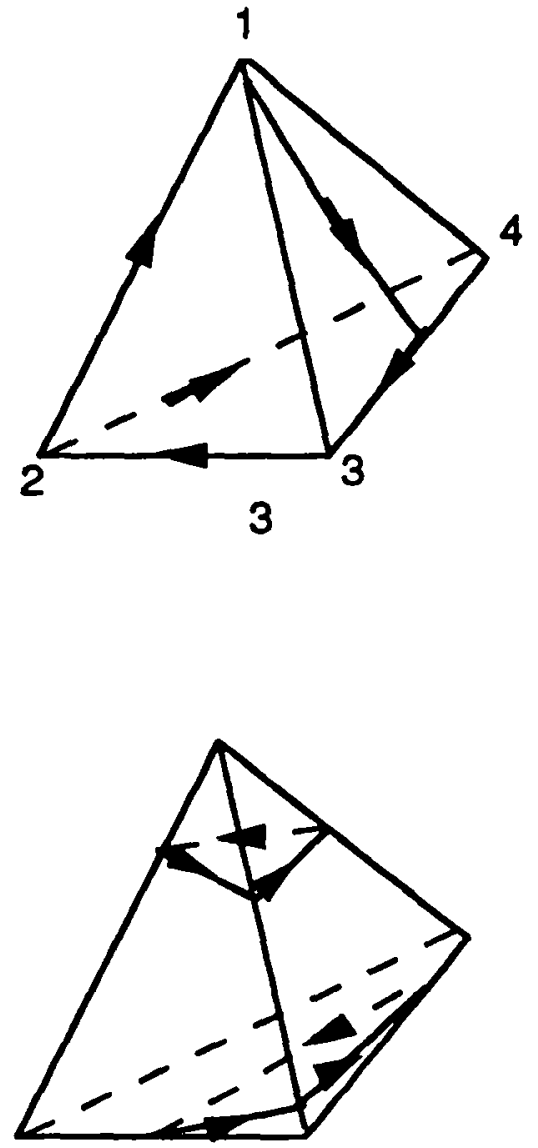

6

Fig. 4.

We choose situations which do not occur in dimension 3.

1. There exists null recurrence on a set of positive measure in the parameter space $\mathbb{P}$. Null recurrent r.w. appear due to scattering. On the fig. 4.1 assume that all $p \Lambda$, dim $\Lambda=$ 3,4 , have nonstable fixed point inside themselves (i.e. $v^{\Lambda}$ have all components negative). For $\operatorname{dim} \Lambda=2, v^{\Lambda}$ are shown on the fig. 4.1. Scattering occurs in the vertices (one-dimensional faces) 1 and 3. Associated Markov chain consists of 6 states - all 1simplexes. It is more convenient to consider all 0 -simplexes as the set of states $\{1,2,3$, 4) with the transition matrix : $p_{23}=p_{42}=1,0<p_{12}, p_{14}, p_{31}, p_{34}<1$, others are zero.

2. Inessential states of the associated Markov chain can give rise to null recurrence. See Fig. 4.2 in the same assumptions as for Fig. 4.1. There states 1,2, 3 are inessential, state 4 is absorbing.

3. On the fig. 4.3 after scattering r.w. goes first along 2-dimensional face 21 and then along the three-dimensional face 134.

4. On the fig. 4.4 an example of possible stable cycle $(3,2,4)$ is given, on Fig. 4.5 possible unstable cycle, two cycles are also possible (see Fig. 4.6). 
General theorem about dimension 4 is the following.

Theorem 2.3.1. There exists a subset $P_{4} \subset \mathbb{P}$ of complete measure such that for this subset : r.w. is recurrent iff $L<0$ for any stable fixed point, any stable cycle and for the associated Markov chain (if there is one).

Theorem 2.3.2. Under the conditions of theorem 2.3.1 let $P_{4,0}$ be the subset of the parameter space for which there are no unstable cycles for the associated dynamical system such that it crosses all four 2-dimensional simplexes (see Fig. 4.5). Then for a subset of complete measure in $P_{4 D}$ r.w. is ergodic iff it is recurrent, $M<0$ for any class of essential states of the associated Markov chain and $M_{\omega}<0$ (see section 4.1.1) for all inessential states $\omega$ of the associated Markov chain. Otherwise it is nonergodic.

The case when there exists an unstable cycle such as on Fig.4.5 is not completely solved. Work is in progress here.

Proofs of these theorems are tedious (see [10]). It consists of the classification of all possible dynamical systems and constructing appropriate Lyapounov functions for different cases. In particular in [10] the subset $\mathbb{P}_{4}$ is explicitly described.

\section{UNIFORM CONVERGENCE}

\subsection{Unique ergodicity}

It is instructive to consider subclasses of $r . w$. for which the following condition holds : for a (closed) union $Q=Q(N)$ of some faces $\Lambda$ of $Z_{+}^{N}$ we have $p_{\alpha \beta}=0$, if $\alpha$ $\in \mathcal{Q}(N), \beta \notin \mathcal{Q}(N)$.

It means that starting from $\mathcal{C}(N)$ one cannot quit it any more. We say in this case that we have a r.w. on $\mathcal{C}(N)$. See [12] where unions of two-dimensional faces are considered.

For a r.w. on $G(N)$ the associated dynamical system is restricted to the subcomplex

$$
P(Q)=\begin{gathered}
\cup P^{\Lambda} \\
\Lambda \subset Q(N)
\end{gathered}
$$

Now we want to consider some cases where continuous invariant measure for associated dynamical system is possible and we get a classification of r.w. in terms of this invariant measure. Concrete examples will be constructed in section 4.1 .

Our first result is

Theorem 3.1.1. Let us consider inductive e.d.r.w. on $Q(N)$. If $T_{t}$ is continuous on $\mathcal{Q}(N)$ and uniquely ergodic then

$$
M=L
$$


moreover r.w. is ergodic if

$$
L<0 \text {. }
$$

Proof. Let us first prove that $L=M$. If $T_{l}$ uniquely ergodic then ( see Theorem 6.19 , $[5]$ ) for any continuous function $g(\omega)$ we have that

$$
\frac{1}{n} \sum_{i=1}^{n} g\left(T^{i} \omega\right)
$$

converges uniformly to a constant $C_{f}$. Let us put now $g=\log f(\omega)$ where $f(\omega)$ is the function defined at the end of the section 1.4. Then $C_{f}=L$.

For any $\varepsilon>0$ there exists $n(\varepsilon)$ such that uniformly in $\omega$

$$
\left|\frac{1}{n} \sum_{i=1}^{n-1} \log f-L\right|<\varepsilon
$$

for $n>n(\varepsilon)$. Then

$$
e^{(L-\varepsilon) n} \leqslant \prod_{i=1}^{n-1} f_{i} \leqslant e^{(L+\varepsilon) n}
$$

So $M=L$ follows.

Now let $L<0$. To prove ergodicity we construct a $L y a p o u n o v$ function for our random walk in the following way. First of all we take the dynamical system $T=T_{t}$ with $t=t$. Note that there exists $k_{0}$ such that

$$
\frac{1}{k} \sum_{i=1}^{k-1} \log f\left(T^{i} \omega\right)<\frac{L}{2}<0
$$

for all $\omega$ and $k>k_{0}$. Any point $x \in \mathrm{R}_{+}^{N}$ can be represented by a pair $(\omega, r)$ where $r=r(x)=\|x\|, \omega=e(x) \in P^{N-1}$.

We take Lyapounov function

$$
F(x)=F(\omega, r)=r
$$

and put

$$
k(x)=\left[k_{0} r\right]+1 \text {. }
$$

having in mind to apply generalised Foster's criteria (see (1) of Appendix)

We want to prove that

$$
E\left(F\left(\xi_{k(x))} / \xi_{0}=(\omega, r)\right)-F(\omega, r) \leqslant-\varepsilon k_{0} r\right.
$$


for all $x=\langle\omega, r\rangle \in \mathbb{Z}_{+}^{N}$ with $r$ sufficiently large. But we know that for $\xi_{k(x)}=$ $\left(\omega_{k}(x), r_{k}(x)\right)$

$$
E r_{k(x)}=r_{0} E \prod_{i=0}^{k(x)-1} f\left(T^{i} \omega\right) \leqslant r_{0} \exp \left(\frac{l}{2}\right)
$$

But from the proposition 1.4.1 we know that for any $\delta>0$ there exists $r_{l}$ such that for $r$ $>r_{1}$

$$
P\left(\left\|\xi_{k(x)}-\left(E_{r_{k}(x)}, T^{k(x)} \omega\right)\right\|>\delta r\right)<\delta
$$

Using boundedness of jumps we get the result .

Let us note that if $G(N)$ is a union of closed faces of the same dimension (say $k$ ) and moreover $T_{t}$ is minimal then only first vector field $M_{A}, \operatorname{dim} \Lambda=k$, plays role. Other details are irrelevant. But in the next theorem one must use $v^{\Lambda}$ instead of $M_{\Lambda}$.

Now let us consider $\mathrm{r}: \mathrm{w}$. on all $\mathbf{z}_{+}^{N}$.

Theorem 3.1.2. Let us assume for some $Q(N)$ that all faces $\Lambda \notin \mathcal{G}(N)$ are ergodic and $w \Lambda$ have all components negative. This means that inside each $P \Lambda, \Lambda \notin Q(N)$, there exists an unstable fixed point of $T_{t}$. Assume that $T_{i}$ on $P(Q)$ (which is invariant w.r.t. $\left.T_{t}\right)$ is uniquely ergodic. Then conclusion of the theorem 3.1 .1 hold.

Proof is similar to the proof of the theorem 3.1.1.

\subsection{Uniformly convergent dynamical systems with many ergodic components}

Let us again consider inductive e.d.r.w. on $\mathrm{G}(\mathrm{N})$.

Theorem 3.2.1. Assume there exist $\delta>0$ and $n_{0}>0$ such that for any $\omega$

$$
\frac{1}{n} \log \prod_{i=0}^{n-1} f\left(T^{i} \omega\right)<-\delta<0
$$

uniformly in $\omega$ and $n>n$. Then r.w. is ergodic.

Proof of this theorem repeats the proof of the theorem 3.1.1.

Example . Let us consider the class $M_{u}$ of dynamical systems $T$ on $Q(N)$ such that for $f(\omega)$

$$
\frac{1}{n} \log \prod_{i=0}^{n-1} f\left(T^{i} \omega\right) \rightarrow L(\omega)
$$

uniformly in $\omega$. If $L(\omega)$ is bounded away from 0 that is 


$$
L(\omega)<-\delta<0
$$

then r.w. is ergodic.

Class $\mathfrak{M}_{u}$ can contain continuum invariant ergodic measures. In fact this theorem comprices many practical cases when the drift to the origin is so strong that other peculiarities do not play any role.

\subsection{Transience for uniformly absorbing attractors.}

Theorem 3.3.1. Under assumptions of theorem 3.1.1 and if $L>0$ then r.w. is transient.

Sketch of the proof. We consider the imbedded Markov chain : $\left\{\xi_{t_{k j}}\right\}$ for some fixed $k$ where $t_{i}$ are all times when $\xi_{t_{i}}$ belongs to nonergodic faces. Then recurrence of the imbedded r.w. is equivalent to the recurrence of the initial r.w. We take $k$ sufficiently large and use supermartingale Foster's criteria (see Appendix, (2)) with the function $\log ^{-1} r$ (or sometimes submartingale criteria with $\log r$ ).

Analog of the theorem 3.1.2 also takes place. Even the more general result is true.

Theorem 3.3.2. Let there exist closed unions of faces $Q_{1}, \ldots, Q_{m}$ of $z_{+}^{N}$ which are mutually disjoint and such that

(i) for any $\Lambda \notin \cup^{m} \mathfrak{Q}_{i}$ all componenets of $\Lambda$ are negative;

$$
i=1
$$

(ii) restriction of $T_{t}$ onto each $\mathcal{Q}_{i}$ is contonuous and uniquely ergodic with an invariant measure $\mu_{i}$;

(iii)

$$
L_{i}=\int g(\omega) d \mu_{i}(\omega) \neq 0 .
$$

Then r.w. is ergodic iff $L_{i}<0$ for all $i$, and is transient otherwise.

Proposition 1.2.3 is a particular case of this theorem when the attractor $\mathcal{Q}_{i}$ consists of one face $\Lambda$ which has an attractive fixed point inside.

\section{MORE DELICATE PHENOMENA}

\subsection{Liaisons dangereuses.}

One must be very courageous to jump (and not to sink) into deep waters of dynamical systems theory not only because of many difficulties but also of seemingly doubtful 
usefulness of such a voyage for practical applications. Nevertheless a general understanding of the world gains more after discoveries of new rich lands and languages. We want to demonstrate now multiple connections with classical dynamical system theory (CDS).

1. CDS usually considers dynamical systems on manifolds. We show that it is also a common case for r.w. in $\mathbf{Z}_{+}^{N}$.

We consider the simplicial complex $P(N, k)=U_{\text {dim } A=k} \overline{P \Lambda}$.

We shall also consider subcomplexes $R \subset P(N, k)$ which are the unions of some $\bar{P} \bar{\Lambda}$ with $\operatorname{dim} \Lambda=k$. Subcomplex $R$ is a compact manifold (which means that any point of $R$ has a neighbourhood homeomorphic to a $k$-dimensional disk) iff any (k-1)-simplex of $R$ belongs to the boundary of exactly two $k$-simplexes of $R$.

Proposition 4.1.1. Let us consider a simplicial complex $S$ which is a compact manifold. Then it is isomorphic to some subcomplex of $P(N, k)$ for some $N$ iff there are no two simplexes of $S$ of some dimension $m>0$ having the same 0 -simplexes.

Proof. Numerate all 0 -simplexes of $S$, take $N$ equal to their number. This will give a correspondence between 0 -simplexes of $S$ and all axis of $R^{N}$.

Class $\mathcal{Q}(N ; R)$. For a subcomplex $R$ of $P(N, k)$ which is a (compact) manifold we consider the class of r.w. for which the following condition takes place:

(i) $\Lambda$ is ergodic iff either $P \Lambda$ does not belong to $R$ or $\operatorname{dim} \Lambda=k$. All components of $v^{\Lambda}$ for all $\Lambda$ with $P \Lambda \nsubseteq R$ are negative.

It follows that any path $\Gamma_{x}$ after a finite time hits $R$ and does not leave it any more. More over it spends all his time on $k$-dimensional simplexes except for denumerable number of moments of time. It is clear that all r.w. in $\mathcal{Q}(N ; R)$ are essentially deterministic. They are also inductive and in many cases their behaviour depends only on ${ }^{\Lambda} \Lambda$ for $\Lambda \subset R$, dim $\Lambda=k$.

2. Continuous transformations (and so topological dynamics) often arises in our case.

Let us consider a r.w. of the class $Q(N, R)$ and the restriction of the corresponding $T_{t}$ onto $R$. Then sufficient condition of its continuity for $\operatorname{dim} R=2$ is the following : for any 0 -simplex $O$ of $R$ there exists exactly one outgoing 2-dimensional face and exactly one ingoing 2-dimensional face, the other 2-dimensional faces of $O$ are neutral with the appropriate directions of the vector field (see Fig. 5 ). 


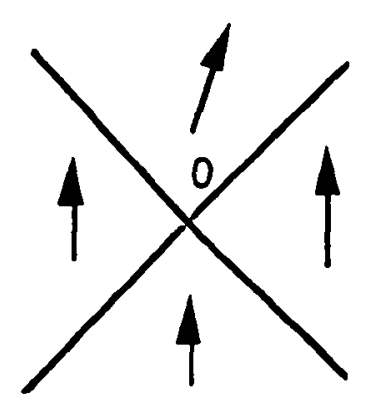

Fig. 5

3. Structural stability and generic properties in our parameter space (small dimensions).

Consider $R$ to be a torus, $\operatorname{dim} R=2$, with the following simplicial structure and vector field (Fig.6).

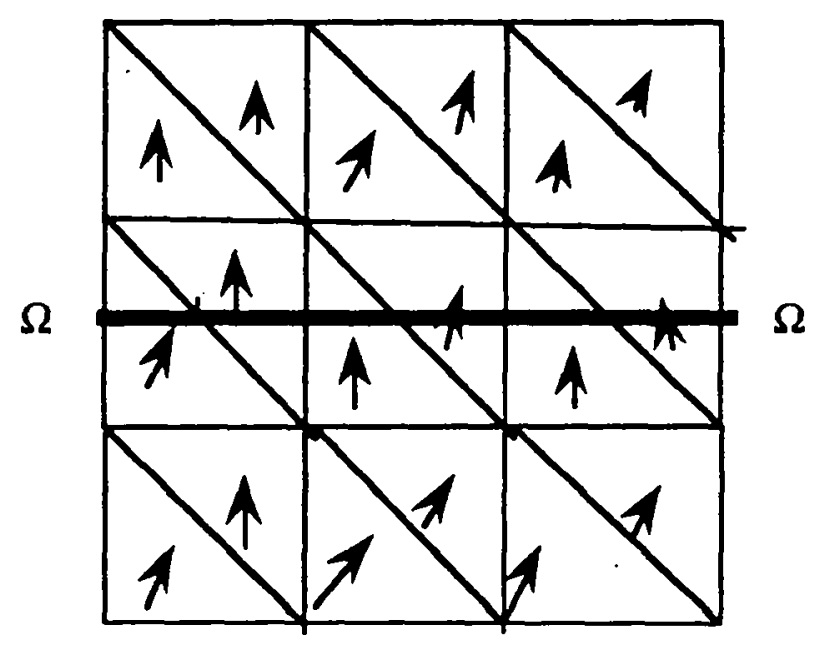

Fig. 6

Let $\Omega=S^{l}$ be a horizontal dissection of the torus (see Fig.6). We consider the dynamical system $(\Omega, U)$, see section 1.4 . We can choose our piecewise linear vector field so that $U$ be a homomorphism (piecewise analytic) of $\Omega$. It is well known that for any such homeomorphism $U$ of a circle its rotation number $\alpha(U)$ is defined.

$P$ is sufficiently meager comparatively e.g. with the space of $C^{1}$-diffeomorphisms. Due to some known results (see [41], p.108) it is reasonable to make a conjecture :

Problem 3.4.1. The set $P(\Omega$, ir) of homeomorphisms of $\Omega$ with irrational (and also rational $\mathbb{P}(\Omega, \mathrm{r})$ ) rotation number have positive measure in $p$.

It is known that for homeomorphisms of $\Omega$ in $P(\Omega$,ir) are uniquely ergodic (see Theorem 5, p. 82, in [4] ). The support of this invariant measure is either all $\Omega$ or a perfect nowhere dense subset of $\Omega$ (see [4]). 
Problem 3.4.1. Does Denjoy's theorem hold for this case? If it does hold then $U$ is topologically equivalent to a rotation and so an invariant measure for homeomorphisms of $\Omega$ in $P(\Omega, i r)$ has $\Omega$ as its support.

Problem 3.4.3. When this measure is absolutely equivalent to Lebesgue measure? This could provide an example (due to theorem 4.1.1) when the ergodicity condition is defined via the integral over this singular measure.

If $\alpha(U)$ is rational and $U$ is structurally stable then the situation is similar to that for the two-dimensional sphere with closed cycles.

\section{Hopeless dimension 5.}

$P(N ; N-1)$ is always a manifold (sphere). So $P(5 ; 4)$ is a manifold homeomorphic to the sphere of dimension 3 . The famous examples $[6,41]$ related to the fact that structurally stable diffeomorphisms are not dense in the space of all diffeomorphisms imply that one cannot hope to obtain complete classification for r.w. in $\mathrm{Z}_{+}^{5}$ under condition $\mathbf{B}_{2}$.

5. Discontinuous maps of an interval. They also appear with positive measure in parameter space. There are many papers on such dynamical systems (see $[33,42]$ ) and it is interesting to establish their connections with r.w. It is not a hopeless problem to obtain the classification of r.w. on 3-dimensional complexes.

\subsection{Null recurrent essentially deterministic random walks : Gibbs measures.}

We know that for small dimensions if r.w. is essentially deterministic then $M=L$. Only for r.w. with scattering phenomena $M \neq L$ occurs. Here we show that $M \neq L$ is very reasonable to be possible also for essentially deterministic r.w. but for dimensions not less than 5 .

Let us consider an inductive e.d.r.w. under condition $B_{2}$ and assume that it gives rise on some compact manifold (as it is explained in the preceding section) to a transitive Anosov diffeomorphism $U$. Let us consider a random perturbation of this diffeomorphism which depends suitably on a small parameter $\varepsilon$ and has a unique invariant measure $\mu_{\varepsilon}$. Then it is known ( see $[21,31,20]$ ) that

$$
\mu_{\varepsilon} \rightarrow \mu(h)
$$

where $\mu(h)$ is an invariant measure for $U$. This invariant measure is the Gibbs measure

$$
\partial \mu(h)(\omega)=\lim _{n \rightarrow-\infty} Z_{n}^{-1}(h) \exp \left(\sum_{i=1}^{n} h\left(U^{i}(\omega)\right) \delta \mu(\omega)\right.
$$


where $\mu$ is the invariant measure for $U$ with maximal entropy (Parry's measure, it is a Markov chain in symbolic dynamics)) which is known to be unique. The function

$$
h(\omega)=-\log \lambda_{u}(\omega)
$$

where $\lambda_{u}(\omega)$ is the differential dilatation coefficient for unstable foliation for $U$. It is known that $h$ belongs to the class of Hölder functions for which Gibbs measure (1) is known to be unique [21] and having nice mixing properties. Let us assume that $g(\omega)=$ $\log f(\omega)$ (see definition 1.5.1) also belongs to this class of Hölder functions. Then the Gibbs measure

$$
\delta \mu(h, g)(\omega)=\lim _{n \rightarrow--} Z_{n}^{-1}(h, g) \exp \left(\sum_{i=I}^{n} g\left(u^{i} \omega\right)\right) d \mu(h)(\omega)
$$

also exists and is equal to $\sigma \mu(h+g)$. So $M$ exists and equals to the free energy

$$
M=F(h, g)=\lim _{n \rightarrow \infty} \frac{1}{n} \log Z_{n}^{-1}(h, g)
$$

and at the same time

$$
L=\int g(\omega) d \mu(h)(\omega)
$$

So we are in the same situation as in [12] where $h=0$ and $f$ is a local function. So similarly to [12] in general $M \neq L$.

Problem 4.2.1. The corresponding r.w. is classified in accordance with Lemma 1.5.2.

\subsection{Continuous exit boundary}

Let us consider a two-dimensional manifold $R \in P(N ; 2)$ and the class $Q(N ; R)$. Let us assume that in a neibourhood of some of its 0 -simplexes $\omega$ we have a vector field shown in Fig.1b.

For any $\omega^{\prime}$ in a neighbourhood of $\omega$ we define $s\left(\omega^{\circ}\right)$ to be the time to reach $\omega^{\prime}$ by moving along reversed dynamical system $T_{-\imath}$. Then $s\left(\omega^{\prime}\right)$ is a continuous function and it is strictly increasing along any ray $R(\omega)$ emanating from $\omega$. It is clear also that the line $O$, $=\left\{\omega^{\prime}: s\left(\omega^{\prime}\right)=s\right\}$ for small $s$ is homeomorphic to the circle. So $\left\{x: x=\Gamma_{\omega}(T\}\right.$ for some $\left.\Gamma_{\omega} \in V_{\omega}\right\}$ is also homeomorphic to the circle. Similarly to the corollary 1.7 .4 we have

Proposition 4.3.1. If the conditions of the proposition 1.4 .3 hold then 
$\min _{\Gamma_{\omega} \in V_{\omega}} \rho\left(\xi_{x(n)}(T n), \Gamma_{\omega n}(T n)\right) \rightarrow 0, n \rightarrow \infty$.

So the circle is the exit boundary for the induced chain similar to one constructed in section 1.7. R.w. tends to one of the points on the boundary depending from which points $x(n)=n \omega_{1}+o(n)$ it starts, $\omega_{1} \neq \omega$ is inside $O_{3}$.

Problem 4.3.1. There exists limiting measure on the circle to which $\xi_{x(n)}(T n)$ tends in distribution.

Neither existence of such limiting measure nor its exact form is not necessary for classification if we never return to this type of the vertex any more (if they are not included to a cycle). We encounter this situation for r.w. in $Z_{+}^{4}$ and also for the above example in the class $\mathcal{Q}(N ; R)$. But it is easy to construct examples of a positive measure in parameter space such vertices are elements of cycles - we come to it as in Fig. 1a and go away from it as in Fig. 1 b. For such cases it is reasonable that these exit boundaries can play role in classification problems.

\subsection{Nonstationary effects and zero drifts.}

We have seen above that in many cases r.w. is classified in terms of the constants $M$ and $L$ which are defined via some invariant measure of a dynamical system (or random process) on a compact space. Natural question arises whether it is always the case except measure zero in parameter space. The answer is negative due to the following example. 1. Sitting too long on innessential states of the associated Markov chain. In [12] it was remarked that if the associated finite Markov chain has inessential states then the constant

$$
M_{\omega}=\lim _{n \rightarrow \infty} \frac{1}{n} \log E_{\omega} \prod_{i=0}^{n-1} f_{i}
$$

where $E_{\omega}$ is the expectation under the condition that we started from the inessential state $\omega$, depends on the initial state $\omega$, so it is not defined by the stationary measure and one must take max over initial states. For an essential state $\omega$ in an irreducible class all $M_{\omega}$ are equal and so defined by stationary distribution but $M_{\omega}$ for inessential state $\omega$ can differ from them.

For nondiscrete $\Omega$ one can easily provide similar examples with not e.d.r.w.

Problem 4.4.1. Provide examples of e.d.r.w. with conditions $A_{12}$ where recurrence is not defined by constant $L$ w.r.t. some invariant measure.

One of the possible ways is to find an unstable invariant set (attractor in the opposite time direction) from which one goes away too slow. It seems quite possible for conditions $B_{2}$, but all existing examples of unstable invariant sets under conditions $A_{12}$ are not such. 
2. Unstable points. They occur when

(i) $v^{A}$ has all components negative. It is common for small dimensions and they are left too quickly to have an influence on the r.w.

(ii) when r.w. is not regular (see Fig. 1 ). This case occurs already in dimension 4 when the vertex 1 of the tetrahedron is unstable. This case is completely described in [10]. No influence on the classification occurs.

3. Unstable cycle. This case also occurs in dimension 4. Equivalently we can consider periodic point on $P(4 ; 1)$ - the set of all 1-dimensional simplexes of the tetrahedron. It is shown in [10] that in dimension 4 unstable cycles do not influence on recurrence or transience of the r.w. up to measure zero in parameter space. It also does not influence on ergodicity except for the case of Fig. 4.5.

4. Zero drifts If in some parts of $\Omega$ one has zero drift ( $v^{\Lambda}=0$ ) the ideology of this paper could survive because of the scaled random suspension. The universality hypothesis however becomes more restricted. It became clear from [11] where dependence on the second moments was exhibited. But nevertheless the answer does not depend on the arithmetical properties of the lattice.

Problem 4.4.1. Provide an example with dependence on the highest moments of the perturbation.

Moreover if for nonzero drifts we have now sufficient intuition this is not the case for zero drifts. Even in dimension 2 where the answer is simple and rigorous proof is obtained we have no yet good understanding of the situation. Previous results on the reflected brownian motion in the angles [45-48] which are closely related to [11] also treat the problem analytically. Our projection onto the simplex $P^{I}$ does not work (Eulers limit is trivial for zero jumps) but as it is common in hydrodynamics one can consider fluctuations (central limit theorem ) and such scaled random suspension seems to be useful.

\subsection{Continuity, analyticity, CLT.}

Almost nothing new about r.w. in $\mathbf{Z}_{+}^{N}$ have appeared after [2] concerning the conditions when ergodic r.w. has stationary probabilities with continuous or analytic dependence on parameters. Only for Jackson networks it was proved in [3]. Related is the question when CLT takes place for r.w. in $\mathbf{z}_{+}^{N}$. It can be proved that CLT holds for analytic Lyapounov Markov chains (see definitions in [3]). 


\section{APPENDIX. ABOUT SOME RELATED GENERAL PROBABILISTIC PROBLEMS}

All criteria used in these problems are essentially of martingale nature. We list them shortly. Let us consider an irreducible aperiodic discrete time Markov chain $\xi_{t}$ with state space $A$ and transition probabilities $p_{\alpha \beta}$. To obtain classification of a chain we use nonnegative function $f(\alpha)$ on $A$ called Lyapounov function .

Ergodicity A chain is ergodic iff there exist $f(\alpha)$, positive integer valued function $k(\alpha)$ on $A$ and $\varepsilon>0$ such that for some finite subset $A_{0} \subset A$

$\sum_{\beta} p_{\alpha \beta}^{k(\alpha)} f(\beta)-f(\alpha) \leqslant-k(\alpha) \varepsilon, \alpha \in A-A_{0}$, and finite for $\alpha \in A_{0}$

It is essentially due to Foster [7] with generalisation in [2], $k(\alpha)$ can be random [27] which is useful in applications. It is necessary and sufficient which means that we can always hope to prove ergodicity but this does not mean that it is useful for to prove nonergodicity.

Transience. A chain is transient iff ( $A_{0}$ is not necessarily finite here ) [7]

$\sum_{\beta} p \alpha \beta^{f(\beta)-f(\alpha)} \leqslant 0, \alpha \in A-A_{0}$,

$\inf \left\{f(\alpha): \alpha \in A_{0}\right\}>\sup \left\{f(\alpha): \alpha \in A-A_{0}\right\}$

Sometimes the following sufficient condition is used :

A chain is transient if there exist $f(\alpha), \sigma>0, A_{0}$ such that

$\sum_{\beta}{ }^{p} \alpha \beta^{f(\beta)-f(\alpha) \geq \varepsilon, \alpha \in A-A_{0},}$

and $p_{\alpha \beta}=0$ if $|\pi(\alpha)-f(\beta)|>d$.

Recurrence. A chain is recurrent iff there exist finite $A_{0}$ and $f(\alpha)$ such that

$\sum_{\beta} p_{\alpha \beta^{f}}(\beta)-f(\alpha) \leq 0, \alpha \in A-A_{0}$, and for all $x$ the set $\{\alpha: f(\alpha)<x\}$ is finite (see [30] ).

Nonergodicity. We have here sufficient condition similar to (3) but with $\varepsilon=0$ and also more powerful sufficient conditions which allow to get necessary and sufficient conditions in two-dimensional zero drift problems, see [11].

There are other criteria but they do not seem to be useful for r.w. in $z_{+}^{N}$.

Problem A1. Get necessary and sufficient conditions for nonergodicity.

If Markov chain is not countable the same criteria exist when all transition conditional distribution are absolutely continuous w.r.t. some common measure. See [28], [37]. It is 
well known however that for Markov chains with mutually singular transition conditional distributions all general classification results fail to be true.

Of course many other methods to prove e.g. ergodicity exist but they can be used either only in very particular cases (majorisation) or only together with other methods (coupling). Natural question arises about the existence of other general methods not related to martingales. They could be useful in zero drift situations.

Further on we consider some probabilistic problems related to r.w. in $z_{+}^{N}$.

\section{Multiplicative suspensions with $L=0$ or $M=0$.}

Let us consider a stationary random process (dynamical system) $\xi_{n}$ with values in a separable metric space $\mathcal{G}$. Let this process live on a probability space $\left(\Omega_{\uparrow}, \Sigma_{\uparrow}, \mu_{1}\right)$.

Let $f(\alpha)$ be a positive function on $Q$ which defines the multiplicative suspension, so $0<$ $a_{1}<f(\omega)<a_{2}, g(\alpha)=\log f(\alpha)$.

For the case when $\xi_{n}$ is a Markov chain the problem is much easier as one can use regeneration property.

Let $\xi_{n}$ be irreducible aperiodic ergodic Markov chain with countable state space $C$. Let $\tau_{1}, \tau_{2}, \ldots$ be all random times when Markov chain is in some fixed state $i$. Let us put $x_{T}=c+\sum_{t=0}^{T-1} g\left(\xi_{t}\right), c>0$.

Then $\eta_{j}=x_{\tau_{j+1}}-x_{\tau_{j}}$ are i.i.d. random variables and $E\left|\eta_{s}\right|$ is finite.

Theorem A1. Under the above conditions multiplicative suspension over $\xi_{n}$ is recurrent.

Proof. We want to prove that $E \eta_{j}=0$.

We shall use notations from [38], p.52, where $i p_{i j}^{(k)}$ is the probability starting from $i$ to reach $/$ after $k$ steps without hitting $/$ any more during these $k$ steps. As $i p / i j=\frac{\pi_{j}}{\pi_{i}}$ we have

$E \eta_{s}=\sum_{k, j} g(j){ }_{i} p_{i j}^{(k)}=\sum_{j} g(j){ }_{i p}^{*}{ }_{i j}^{*}=\sum_{j} g(j) \frac{\pi_{j}}{\pi_{i}}=0$

Then we have that $x_{\tau_{i}}$ enters 0 a.s. and so $x_{n}$ does. 
Similar result could be proved for ergodic Markov chains with absolutely continuous (w.r.t. some measure) transition probabilities. See [43] for related results. Unfortunately I do not know more general conditions under which theorem Al holds.

Let us assume the following wittingly exagerated mixing conditions :

there exist $0<\gamma<1, C>0$, such that for any bounded real functions $f(\alpha), \alpha \in \mathbb{Q}$, any $t_{f}<t_{2}<\ldots<t_{n}$ and positive integers $k_{1}, \ldots, k_{n}$ the semiinvariants have "uniform exponential decay" (see [36] for notations and terminology):

$\left|\left\langle f_{I}^{k_{1}}\left(\xi_{t_{I}}\right), \ldots, r_{n}^{k_{n}}\left(\xi_{t_{n}}\right)\right\rangle\right| \leqslant \gamma^{n-t_{1}} \Pi_{i} k_{i} !\left(C \sup \left|f_{i}\right|\right)^{k_{i}}$

Problem A1. Prove that under conditions (1) and $L=O$ the multiplicative suspension is recurrent.

For assumption (1) it is the well known fact for one-dimensional Gibbs random fields that $M$ always exists and moreover when $M=0$

$$
E \prod_{i=0}^{n-1} f\left(\xi_{i}\right) \rightarrow \text { const ., } n \rightarrow \infty
$$

Problem A2. Prove that under conditions (1) and $M=0$ the multiplicative suspension is nonergodic. In fact I know only two methods to prove that mean return time is infinite : analytic methods for independent r.v. and construction of appropriate martingale in a general case. It is an exercise to construct such a martingale for the case when $\xi_{t}$ is a finite Markov chain. It is an interesting problem to provide an alternative approach. 


\section{CONCLUSION}

1. We considered r.w. in $\mathbf{Z}_{+}^{N}$ or networks with identical customers and have shown that in many cases their classification is possible only if we have sufficient control over the associated dynamical system on the compact set and its random perturbation. Moreover any smooth dynamical system is the associated dynamical system for some r.w. in $z_{+}^{N}$. So the complexity of classification of r.w. is comparable with that for general ergodic theory.

2. Deep results from ergodic theory can be used in some r.w. problems. In general in a r.w. problem one should study first the associated dynamical system and using it construct an appropriate Lyapounov function.

3. Associated dynamical systems could be used for obtaining and foreseeing classification results and also for numerical modelling. In general only two real constants $M$ and $L$ define the classification. This fact can simplify the modelling considerably.

4. The notion of the scaled random perturbation is introduced which can have an independent interest. 


\section{REFERENCES}

1F. Bacelli, A.M. Makovski . Queueing models for systems with synchronization constraints. Proceedings of the IEEE,v.77,No.1,1989, 138-161.

2.VA. Malyshev,M.V. Menshikov.Ergodicity,continuity and analyticity of countable Markov chains. Trans.Moscow. Math. Soc.,1979,v.39,pp.3-48.

3. G. Fayolle,V.A. Malyshev, M.V. Menshikov, A.F. Sidorenko.Lyapounov functions for Jackson networks.Rapport de Recherche INRLA, 1991, no.1380.

4. I.P. Kornfeld,Ya.G. Sinai,S.V. Fomin. Ergodic theory.1980. Moscow.

5. P. Walters. An Introduction to Ergodic Theory.Springer Verlag.1975.

6. R. Mañé. Ergodic theory and Differentiable Dynamics.Springer Verlag.1987.

7. Foster F.G. On stochastic matrices associated with certain dueueing processes. Ann.Math. Stat.,24 (1953), 355 - 360.

8.V.A. Malyshev. Classification of two-dimensional random walks and almost linear semimartingales. Dokl. Akad.Nauk USSR,1972,202,no.3,526-528.

9. M.V. Menshikov. Ergodicity and transience conditions for random walks in the positive octant of space. Dokl.Akad.Nauk SSSR, 217 (1974) , 755 - 758.

10. I.A. Ignatyuk, V.A. Malyshev. Classification of random walks in $z_{+}^{4}$. INRIA preprint. 1991.

11. G. Fayolle,V.A. Malyshev, M.V. Menshikov.Randoms walks in a quarter plane with zero drift.1 : ergodicity and null recurrence. Rapport de Recherche INRIA, 1990, no. 1314, pp.1-20 (with ). Submitted to Annales d'Institut Henri Poincare.

12. G. Fayolle,V.A. Malyshev, IA. Ignatyuk, M.V. Menshikov. Random walks in two dimensional complexes. Rapport de Recherche INRIA, 1990. To appear in "Queueing theory",1991.

13. W. Szpankowski.Towards computable stability criteria for some multidimensional stochastic processes. In "Stochastic Analysis of Computer and Communication Systems". H.Takagi (Editor). 1990. North-Holland.

14. Botvich $D$., Zamyatin A. Lyapounov functions for some BCMP and Kelly networks. Unpublished.

15. Rybko A.N. , Stolyar A.L. On the problem of ergodicity of Markov processes corresponding to the message switching networks. Preprint.

16. Has'minskii , R.Z. Necessary and sufficient conditopns for the asymptotic stability of linear stochastic systems. Theory Probab. Applic. 12 (1967), 144 - 147.

17. Has'minskii , R.Z. Stochastic stability of differential equations. Alphen: Sijthoff and Noordhoof. 1980. 
18. L. Arnold, E. Oeljeklaus, E. Pardoux. Almost sure and moment stability for linear Ito equations. In "Lyapunov exponents", Lect. Notes Math. , v. 1186, pp. 129-159. 19. D. Ruelle. Thermodynamic formalism. Addison-Wesley Pub.Com.1978.

20. Young L.S. Stochastic stability of hyperbolic attractors. Ergodic theory and Dynam.Syst.,1986, v.6, pp. 311-319.

21. Sinai Ya.G. Gibbs measures in ergodic theory. Uspekhi Mat. Nauk,1972, v. 27 , No.4, pp. 21-64.

22. M.I. Freidlin, A.D. Wentzell. Random perturbations of dynamical systems.1984. Springer Verlag.

23. Yu. Kifer.Ergodic theory of random transformations. 1986. Birkhäuser.

24. W. Feller. Boundaries induced by positive matrices. Trahs. Amer. Wath. Soc., vol.83 (1956),pp. 19-54.

25. J.L. Doob. Discrete potential theory and boundaries. J. Math. Mechanics, vol.8, (1959),pp. 433-458.

26. VI. Oseledec. Multiplicative ergodic theorem. Characteristic Lyapounov exponents of dynamical systems. Trudy Moscow Math. Soc.,1968, v. 19, 179-210.

27. Filonov Yu.P. Ergodicity criteria for homogeneous discrete Markov chains. Ukrainian Math. J., 1989, v.41, No. 10, 1421-1422.

28. A.A. Borovkov. Lyapounov functions and ergodicity of multidimensional Markov chains. Institute of Mathematics of Sibirian Ac. of Sci. Preprint No. 19. Novosibirsk. 1990.

29. V.A. Mikhailov. Geometric analysis of stability of Markov chains in $\mathbb{R}_{+}^{N}$ and its applications. Problems of information transmission, 1988, v.24, No.1, 61-73. 30. Mertens J.-F., Samuel-Cahn E., Zamir S. Necessary and sufficient conditions for recurrence and transience of Markov chains in terms of inequalities. J. Appl. Prob., 1978, v. 15, pp. 848-851.

31. Yu. Kifer. General random perturbations of hyperbolic and expanding transformations. J. d'Analyse Mathématique, v.47 (1986), 11-150.

32. Yu. Kifer. Principal eigenvalues, topological pressure and stochastic stability of equilibrium states. Israel J. of Math., v. 70, No. 1, 1990; 1-47.

33. A. Katok, Yu. Kifer. Random perturbations of transformations of an interval. J. d'Analyse Mathématique, v.47 (1986), 193-237.

34. V.A. Malyshev. Random walks.The Wiener-Hopf equations in a quadrant of the plane.Galois automorphisms.Moscow State University Press. Moscow,1970,201 pp.

35. G. Fayolle, R. Iasnogorodski. Two coupled processors : the reduction to a Riemann - Hilbert problem. Z. Wahrsch. verw. Gebiete, 1979, 47, 325-351.

36. V.A. Malyshev, R.A. Minlos. Gibbs random fields. Method of cluster expansions. Moscow. 1985. (English translation in Kluwer, 1991 ). 
37. Tweedie R.L. Criteria for classifying general Markov chains. Adv. Appl. Prob., 8, 1976, 737-771.

38. Chung $K L$. Markov chains with stationary transition probabilities. 1967. Springer.

39. Kelly F.P. Loss networks. Cambridge Univ. Preprint. 1990.

40. M. Kelbert, Yu. Suhov. Mathematical problems of queueing networks. In "Probability theory.Mathematical. Statistics. Theoretical cybernetics. Itogi nauki i techniki",Akad.Nauk SSSR, Vsesoyuz. Inst. Naucn. i Techn.Informacii,Moscow,1988, v.26, 3-96.

41. VI. Arnold. Geometrical methods in the theory of ordinary differential equations. Springer. 1983.

42. J. Willms. Asymptotic behaviour of iterated piecewise monotone maps. Erg. Theory and Dyn. Systems, 1988, 8, 111-131.

43. Bougerol $P$. Oscillation de produits de matrices alèatoires dont l'exposant de Lyapunov est nul. In "Lyapunov exponents", Lect. Notes Math. , v. 1186, pp. 27-36.

44. Blank M.L. Small perturbations of haotic dynamical systems. Uspehi Mat. Nauk., 1989 , v. 44, No.6, 3-28.

45. M.I. Reiman, R.J. Williams. A boundary property of semimartingale reflecting brownian motions. Prob. Theor. Rel. Fields, 1988, v. 77, 87-97.

46. S.R.S. Varadhan, R.J. Williams. Brownian motion in a wedge with oblique reflection. Commun. on Pure and Applied Mathematics, 1985, v. 38, 405-443.

47. R.J. Williams. Recurrence classification and invariant measure for reflected brownian motion in a wedge. Ann. Prob., 1985, v. 13, No. 3, 758-778.

48. I.L. Genis, N.V. Krylov. About exact barriers in the problem with oblique derivative. Sibirian Math. J. 1973, v. 14, No. 1, 36-43. 
ISSN $0249-6399$ 